\title{
Modeling Atmospheric Turbulence via Rapid Distortion Theory: Spectral Tensor of Velocity and Buoyancy
}

\author{
Chougule, Abhijit S.; Mann, Jakob; Kelly, Mark C.; Larsen, Gunner Chr.
}

Published in:

Journal of the Atmospheric Sciences

Link to article, DOI:

10.1175/JAS-D-16-0215.1

Publication date:

2017

Document Version

Publisher's PDF, also known as Version of record

Link back to DTU Orbit

Citation (APA):

Chougule, A. S., Mann, J., Kelly, M. C., \& Larsen, G. C. (2017). Modeling Atmospheric Turbulence via Rapid Distortion Theory: Spectral Tensor of Velocity and Buoyancy. Journal of the Atmospheric Sciences, 74(4), 949974. https://doi.org/10.1175/JAS-D-16-0215.1

\section{General rights}

Copyright and moral rights for the publications made accessible in the public portal are retained by the authors and/or other copyright owners and it is a condition of accessing publications that users recognise and abide by the legal requirements associated with these rights.

- Users may download and print one copy of any publication from the public portal for the purpose of private study or research.

- You may not further distribute the material or use it for any profit-making activity or commercial gain

- You may freely distribute the URL identifying the publication in the public portal 


\title{
${ }^{`}$ Modeling Atmospheric Turbulence via Rapid Distortion Theory: Spectral Tensor of Velocity and Buoyancy
}

\author{
ABhiJit Chougule \\ Department of Engineering Sciences, University of Agder, Grimstad, Norway \\ JAKOB MANN, MARK KELly, AND GUNNER C. LARSEN \\ Wind Energy Department, Technical University of Denmark, Ris $\varnothing$ Campus, Roskilde, Denmark
}

(Manuscript received 22 July 2016, in final form 21 November 2016)

\begin{abstract}
A spectral tensor model is presented for turbulent fluctuations of wind velocity components and temperature, assuming uniform vertical gradients in mean temperature and mean wind speed. The model is built upon rapid distortion theory (RDT) following studies by Mann and by Hanazaki and Hunt, using the eddy lifetime parameterization of Mann to make the model stationary. The buoyant spectral tensor model is driven via five parameters: the viscous dissipation rate $\varepsilon$, length scale of energy-containing eddies $L$, a turbulence anisotropy parameter $\Gamma$, gradient Richardson number (Ri) representing the local atmospheric stability, and the rate of destruction of temperature variance $\eta_{\theta}$. Model output includes velocity and temperature spectra and associated cospectra, including those of longitudinal and vertical temperature fluxes. The model also produces two-point statistics, such as coherences and phases of velocity components and temperature. The statistics of uniformly sheared and stratified turbulence from the model are compared with atmospheric observations taken from the Horizontal Array Turbulence Study (HATS) field program, and model results fit observed one-dimensional spectra quite well. For highly unstable stratification, however, the model has deficiencies at low wavenumbers that limit its prediction of longitudinal velocity component spectra at scales on the order of $0.6 \mathrm{~km}$. The model predicts coherences well for horizontal separations but overestimates vertical coherence with increasing separation. Finally, it is shown that the RDT output can deviate from Monin-Obukhov similarity theory.
\end{abstract}

\section{Introduction}

Turbulent motions, as a result of their fundamentally chaotic and irregular nature, are typically treated statistically rather than deterministically. In the atmospheric boundary layer (ABL), the Reynolds number is typically very large $\left(\mathrm{Re} \sim 10^{6}-10^{8}\right.$; cf. Wyngaard 2010$)$, so the wind in the ABL is nearly always turbulent. The mechanisms for generating and maintaining turbulence in the atmosphere include (mean) shear and buoyant production, where the latter is typically due to heating or cooling of the ground; a description of how the ABL responds to changes in atmospheric stability may be

Denotes content that is immediately available upon publication as open access.

Corresponding author e-mail: A. Chougule, abhijit.chougule@ uia.no found in, for example, Kaimal and Finnigan (1994) or Wyngaard (2010).

Spectra of velocity fluctuations in the ABL range from millimeters to kilometers spatially and temporally from fractions of a second to hours. Spectra and crossspectra of wind speed measurements vary depending on atmospheric stability, which affects them particularly at low frequencies (Chougule et al. 2015). In addition to the velocity components, the temperature acts as an active scalar by modifying the velocity field, particularly the vertical component, through buoyancy.

The spectral characteristics of surface-layer turbulence have previously been studied using Monin-Obukhov similarity theory (MOST) based on dimensional analysis and empirical relationships derived from experiment (Businger et al. 1971; Kaimal et al. 1972; Wyngaard and Coté 1972; Wyngaard 2010). It is established that highfrequency and smaller-scale $(<\sim 100 \mathrm{~m})$ motions follow the Kolmogorov power law, where the velocity and temperature spectra decay with frequency as $f^{-5 / 3}$, 
whereas the slope of the cospectrum of longitudinal $(u)$ and vertical $(w)$ velocity and the cospectrum between $w$ and potential temperature $\theta$ follows $f^{-7 / 3}$ in the inertial subrange. The power-law behavior of inertial-range $u \theta$ cospectra is not as well known, with multiple values noted in Kaimal et al. (1972).

The spatial characteristics of homogeneous neutral ABL turbulence were modeled through a stationary spectral velocity tensor in Mann (1994b, hereafter M94). It incorporates rapid distortion theory (RDT; Townsend 1976; Pope 2000), which involves linearization of the Navier-Stokes equations (NSE) in Fourier space, plus assumption of uniform mean shear and a wavenumberdependent eddy lifetime. The spectral shapes and coherences of the velocity fluctuations that the model predicts have previously been compared with data measured over sea, over flat rural terrain, and even in boundary layer wind tunnels (Mann 1994a). Although the model was not intended to account for buoyancy effects, it has been used to describe one-point spectra in nonneutral conditions as in Peña et al. (2010a,b) and Sathe et al. (2013). The model performance was validated and compared against different roughness lengths in Chougule et al. (2015) and by de Maré and Mann (2014) for offshore winds. RDT had previously been used in nonstationary spectral tensor modeling of homogeneous uniformly sheared (Maxey 1982), unsheared stably stratified (Hanazaki and Hunt 1996), and sheared stably stratified (Hanazaki and Hunt 2004, hereafter HH04) turbulent flows. Recently, de Maré and Mann (2016) further investigated spectral tensor modeling of the neutral ABL, also including temporal fluctuations, by modeling velocity correlations in time as well as in space.

Heidrick et al. (1977) measured axial velocities at two points in water in fully developed pipe flow at various radial positions, and the phases of the cross-spectra of these velocities were given in terms of a wave model of turbulence. With a separation vector that is oriented at different angles to the mean flow, they show that the loci of equal phase are inclined to the mean flow and the inclination increases with distance from the wall. However, a twopoint cross-spectral study in Chougule et al. (2012) suggests that the arrival time shifts of turbulence at two heights are different for the three turbulent velocity components, and that would give different equal-phase loci per component. Later, both through the experiment and a nonstationary spectral model, Komori et al. (1983) studied the phase angle between the vertical velocity component and the temperature in stably stratified open-channel flow. Komori et al. (1983) had a similar approach to ours, but (as in M94) we employ the concept of eddy lifetime to make the model stationary, whereas Komori et al. (1983) terminate integration of their spectral solutions at a nondimensional terminal time, which they determined to be equal to 4 time units from the comparisons with measurements in neutral flow. Further, a value between 3 and 6 units of magnitude of mean velocity gradient multiplied by a time scale (equal to TKE/ $\varepsilon$, where TKE is the specific turbulent kinetic energy and $\varepsilon$ is the rate of viscous dissipation of TKE) was noted in Pope (2000). Another difference in our approach is that we consider a nonzero initial temperature fluctuation. The spatial wavenumber-dependent correlations and the arrival-time shift in the temperature fluctuations between two points in space are missing in the studies mentioned above. In this paper, we investigate such aspects in terms of squared coherence and crossspectral phases for both stable and unstable stratification. For the simulation of turbulence and subsequent estimation of its loading effects upon structures in the lower atmosphere, it would be useful to augment the spectral tensor model (M94) to include buoyancy effects and to predict the temperature fluxes. The paper starts with mathematical modeling, as described in section 2. Section 3 provides information about the Horizontal Array Turbulence Study (HATS) experiments and the method used for the model validation. The results from the modeling and observations are discussed in section 4 .

\section{Spectral tensor modeling by RDT}

Here we describe the basic properties of the spectral tensor, the governing RDT equations with assumptions, and the initial conditions as the state of the isotropic turbulence, including the von Kármán energy spectrum (von Kármán 1948).

\section{a. Definitions and properties}

We consider a three-dimensional fluctuating velocity field $\mathbf{u}^{\prime}(\mathbf{x}, t)$, where $\mathbf{x}=\{x, y, z\}$ is the (longitudinal, lateral, and vertical) position vector in space. The spectral representation of $\mathbf{u}^{\prime}(\mathbf{x}, t)$, to be considered as nonperiodic, can be given in terms of the Fourier-Stieltjes integral (Wyngaard 2010):

$$
\mathbf{u}^{\prime}(x)=\int e^{i \mathbf{k} \cdot \mathbf{x}} d \mathbf{Z}(\mathbf{k}),
$$

where the integration is over all wavenumber space (Batchelor 1953), and $\mathbf{k}=\left(k_{1}, k_{2}, k_{3}\right)$ is the wavenumber vector. Since the mean of the fluctuations, $\overline{\mathbf{u}^{\prime}}(\mathbf{x}, t)$, is zero, it follows from (1) that the mean $\overline{d \mathbf{Z}}(\mathbf{k}, t)$ is also zero. Also, since $\mathbf{u}^{\prime}(\mathbf{x}, t)$ is a real vector, for each $\mathbf{k}$, $d \mathbf{Z}(\mathbf{k}, t)$ is a complex vector that satisfies conjugate symmetry:

$$
d \mathbf{Z}(\mathbf{k}, t)=d \mathbf{Z} *(-\mathbf{k}, t),
$$


where an asterisk denotes the complex conjugate, and continuity $\left(\nabla \cdot \mathbf{u}^{\prime}=0\right)$ implies that $d \mathbf{Z}$ is normal to $\mathbf{k}$ : that is,

$$
\mathbf{k} \cdot d \mathbf{Z}=0
$$

Given that $\overline{d \mathbf{Z}}(\mathbf{k}, t)=0$, we consider a second-order statistic - the covariance of two Fourier coefficients, $\left\langle d Z_{i}\left(\mathbf{k}^{\circ}, t\right) d Z_{j}(\mathbf{k}, t)\right\rangle$-or, in terms of the covariance tensor,

$$
R_{i j}\left(\mathbf{x}^{\circ}, \mathbf{r}, t\right)=\left\langle u_{i}^{\prime}\left(\mathbf{x}^{\circ}, t\right) u_{j}^{\prime}\left(\mathbf{x}^{\circ}+\mathbf{r}, t\right)\right\rangle .
$$

Here the angle brackets denote the ensemble average operator. It can be shown that the covariance of two Fourier coefficients is the Fourier transform of the covariance tensor and that, for homogeneous turbulence,

$$
R_{i j}(\mathbf{r}, t)=R_{j i}(-\mathbf{r}, t)
$$

In homogeneous turbulence, the spectral velocity tensor is defined as

$$
\Phi_{i j}(\mathbf{k})=\frac{1}{(2 \pi)^{3}} \int R_{i j}(\mathbf{r}) e^{-i \mathbf{k} \cdot \mathbf{r}} d \mathbf{r},
$$

where $\int d \mathbf{r} \equiv \int_{-\infty}^{\infty} \int_{-\infty}^{\infty} \int_{-\infty}^{\infty} d r_{1} d r_{2} d r_{3}$, and the time dependence has been left out for simplicity. As shown in (6), $\Phi_{i j}(\mathbf{k})$ and $R_{i j}(\mathbf{r})$ form a Fourier-transform pair. The two-point correlations of the Fourier velocity components are related to the spectral tensor by

$$
\frac{\left\langle d Z_{i}^{*}(\mathbf{k}) d Z_{j}(\mathbf{k})\right\rangle}{d k_{1} d k_{2} d k_{3}}=\Phi_{i j}(\mathbf{k}) .
$$

The spectral tensor $\Phi_{i j}(\mathbf{k})$ is a complex quantity that has the properties

$$
\begin{aligned}
\Phi_{i j}(\mathbf{k}) & =\Phi_{j i}^{*}(\mathbf{k})=\Phi_{j i}(-\mathbf{k}) \quad \text { and } \\
k_{i} \Phi_{i j}(\mathbf{k}) & =k_{j} \Phi_{i j}(\mathbf{k})=0,
\end{aligned}
$$

which can be interpreted from (2) and (3).

The spectral tensor contains information about the second-order statistics of the three velocity components through the indices $\{i, j\}$. For example, setting $\mathbf{r}=0$ in (6), we obtain the one-point statistics:

$$
R_{i j}(0)=\left\langle u_{i}^{\prime} u_{j}^{\prime}\right\rangle=\int \Phi_{i j}(\mathbf{k}) d \mathbf{k},
$$

where $\Phi_{i j}(\mathbf{k})$ represents the Reynolds stress "density" in wavenumber space. The specific turbulent kinetic energy is equal to half the trace of the matrix above: $\mathrm{TKE} \equiv(1 / 2)\left\langle u_{i}^{\prime} u_{i}^{\prime}\right\rangle=(1 / 2) R_{\ddot{i}}(0)$. The directional information in $\mathbf{k}$ can be removed by integrating over wavevectors with magnitude $k=|\mathbf{k}|$, resulting in a "scalar" TKE spectrum $E(k)$ that also has the property

$$
\mathrm{TKE}=\int_{0}^{\infty} E(k) d k,
$$

where

$$
E(k)=\int_{|\mathbf{k}|=k} \frac{1}{2} \Phi_{i i}(\mathbf{k}) 4 \pi k^{2} d k .
$$

The spectral representation of the cross correlation of the pair of stochastic processes separated by $\mathbf{r}=(0, \Delta y, \Delta z)$ is given as the set of all cross-spectra:

$\chi_{i j}\left(k_{1}, \Delta y, \Delta z\right)=\frac{1}{2 \pi} \int_{-\infty}^{\infty} R_{i j}(x, \Delta y, \Delta z) e^{-i k_{1} x} d x$,

which is of significant importance in practical applications, such as estimation of loads on structures. The relationship between the components of the spectral tensor and the cross-spectra is

$$
\chi_{i j}\left(k_{1}, \Delta y, \Delta z\right)=\int \Phi_{i j}(\mathbf{k}) e^{i\left(k_{2} \Delta y+k_{3} \Delta z\right)} d \mathbf{k}_{\perp},
$$

where $\int d \mathbf{k}_{\perp} \equiv \int_{-\infty}^{\infty} \int_{-\infty}^{\infty} d k_{2} d k_{3}$, and $\Delta y$ and $\Delta z$ are transverse and vertical separations, respectively. Using (13), the single-point power spectrum of the $\iota$ th velocity component (where $\Delta y=\Delta z=0$ ) can be given as $F_{\iota}\left(k_{1}\right)=\chi_{\iota \iota}\left(k_{1}, 0,0\right)$, where the Greek index $\iota$ indicates no summation over indices.

The cross-spectral properties can be expressed in terms of squared coherence (coh) and the cross-spectral phase $\varphi$ as

$$
\begin{aligned}
\operatorname{coh}_{\iota \lambda}\left(k_{1}, \Delta y, \Delta z\right) & =\frac{\left|\chi_{\iota \lambda}\left(k_{1}, \Delta y, \Delta z\right)\right|^{2}}{F_{\iota}\left(k_{1}\right) F_{\lambda}\left(k_{1}\right)}, \\
\varphi_{i j}\left(k_{1}, \Delta y, \Delta z\right) & =\arg \left[\chi_{i j}\left(k_{1}, \Delta y, \Delta z\right)\right] .
\end{aligned}
$$

Following the above discussion, the spectral tensor is modeled using (7), where the equation for the evolution of the Fourier modes $d \mathbf{Z}(\mathbf{k}, t)$ is deduced from NSE via RDT. The indices $i, j=\{1,2,3\}$ are used for the velocity components-that is, $\left\{u_{1}^{\prime}, u_{2}^{\prime}, u_{3}^{\prime}\right\}=\left\{u^{\prime}, v^{\prime}, w^{\prime}\right\}$-and for the space vector $\left\{x_{1}, x_{2}, x_{3}\right\}=\{x, y, z\}$. In later sections, where the (scalar) buoyancy variable is also included, we will use the index notation $l, m=\{1,2,3,4\}$ for the three velocity components along with the temperature fluctuations. For easier-to-read presentation, we use overbars for the mean of a velocity component or scalar, whereas we use angle brackets for higher-order statistics, though by definition both notations have the same meaning 
(i.e., $\overline{u^{\prime}}=\left\langle u^{\prime}\right\rangle$ ). In the next section, we provide the Fourier transform of the NSE, where we use the assumption of homogeneity, and the RDT limit for the modeling via (7).

\section{b. Governing equations: Assumptions and RDT limits}

The governing NSE of the turbulent velocity field $\mathbf{u}(\mathbf{x}, t)$ can be written as

$$
\frac{\partial u_{i}}{\partial t}+u_{j} \frac{\partial u_{i}}{\partial x_{j}}=-\frac{1}{\rho} \frac{\partial p}{\partial x_{i}}+\nu \frac{\partial^{2} u_{i}}{\partial x_{j} \partial x_{j}}-\delta_{i 3} g,
$$

where $u_{i}$ is the instantaneous $i$ th velocity component, $p$ is the pressure, $\rho$ is the density, $\nu$ is the kinematic viscosity, and $g$ is the acceleration due to gravity. Here we neglect the Coriolis force, following M94. The flow field $\mathfrak{\Im}$ can be described into the mean and the fluctuating part by Reynolds decomposition:

$$
\mathfrak{I}=\overline{\mathfrak{I}}+\mathfrak{\Im}^{\prime} .
$$

Considering hydrostatic balance (where the vertical-mean pressure gradient is balanced by the specific weight of the fluid), neglecting second-order fluctuations and viscosity, and assuming horizontal homogeneity, the equation of motion for the $i$ th fluctuating velocity component can be obtained from (17) as

$$
\frac{D u_{i}^{\prime}}{D t}=-\frac{1}{\bar{\rho}} \frac{\partial p^{\prime}}{\partial x_{i}}+\delta_{i 3} g \frac{\theta^{\prime}}{\bar{\theta}}-u_{j}^{\prime} \frac{\partial \bar{u}_{i}}{\partial x_{j}},
$$

where $D / D t \equiv \partial / \partial t+\bar{u}_{j} \partial / \partial x_{j}$ is the total derivative. We often consider the mean velocity field,

$$
(\bar{u}, \bar{v}, \bar{w})=(U, 0,0) .
$$

Equation (19) contains a buoyancy term, which is the second term on the right-hand side of the equation. The density of dry air can be calculated using the equation of state of an ideal gas; $p=\rho R T$, where $R$ is the specific gas constant, $T$ is absolute temperature, and the relationship $T^{\prime} / \bar{T}=\theta^{\prime} / \bar{\theta}$ (Wyngaard 2010) has been used in (19).

Neglecting molecular diffusivity, assuming horizontal homogeneity, and neglecting nonlinear terms, via (18) and (20) the potential temperature equation is given as

$$
\frac{D \theta^{\prime}}{D t}=-w^{\prime}\left(\frac{d \bar{\theta}}{d z}\right),
$$

where $\partial \bar{\theta} / \partial z \equiv d \bar{\theta} / d z$ is the uniform potential temperature gradient (lapse rate). The mean potential temperature can be expressed as $\bar{\theta}=\bar{T}+\left(g / c_{p}\right) \delta z$, where $\delta z$ is the height difference from the 1000-hPa level, $c_{p} \approx$ $1005 \mathrm{~J} \mathrm{~kg}^{-1} \mathrm{~K}^{-1}$ is the specific heat at constant pressure, and $d \bar{\theta} / d z=\partial \bar{T} / \partial z+g / c_{p}$. By neglecting the specific humidity, (21) takes into account the influence of a uniform lapse rate, to be determined.

The pressure term in (19) is further represented in terms of the velocity field by taking the divergence of (17) and using Reynolds decomposition according to (18), resulting in the Poisson equation

$-\frac{1}{\bar{\rho}} \nabla^{2} p^{\prime}=2 \frac{\partial \bar{u}_{j}}{\partial x_{i}} \frac{\partial u_{i}^{\prime}}{\partial x_{j}}+\frac{\partial^{2}}{\partial x_{i} \partial x_{j}}\left(u_{i}^{\prime} u_{j}^{\prime}-\left\langle u_{i}^{\prime} u_{j}^{\prime}\right\rangle\right)-\frac{g}{\bar{\theta}} \frac{\partial \theta^{\prime}}{\partial z}$.

The consequence of introducing a buoyancy term in the momentum equation is the last term in the above equation. The Poisson equation for $p^{\prime}$ consists of terms that represent interaction between the turbulent field and mean velocity gradient [first term on right-hand side of (22)], turbulence-turbulence interaction (second term), and the effect due to the uniform lapse rate through the gradient of potential temperature fluctuation (last term). In the rapid distortion limit, we assume that the first term on the right-hand side of the equation is much larger than the second term, so (22) simplifies to

$$
-\frac{1}{\bar{\rho}} \nabla^{2} p^{\prime}=2 \frac{\partial \bar{u}_{j}}{\partial x_{i}} \frac{\partial u_{i}^{\prime}}{\partial x_{j}}-\frac{g}{\bar{\theta}} \frac{\partial \theta^{\prime}}{\partial z} .
$$

Using the Fourier-Stieltjes integral in (1) for velocity, pressure, and potential temperature fluctuations, we Fourier transform (19), (21), and (23) respectively. We assume homogeneity of the fluctuations, include the influence of the mean uniform shear, and assume that $d \bar{\theta} / d z$ does not change with $z$, to get

$$
\begin{aligned}
\frac{D}{D t} d Z_{i}[\mathbf{k}(t), t]= & \left(2 \frac{k_{i} k_{1}}{k^{2}}-\delta_{i 1}\right)\left(\frac{d U}{d z}\right) d Z_{3}[\mathbf{k}(t), t] \\
& -\frac{g}{\bar{\theta}}\left(\frac{k_{i} k_{3}}{k^{2}}-\delta_{i 3}\right) d \Theta[\mathbf{k}(t), t]
\end{aligned}
$$

and

$$
\frac{D}{D t} d \Theta[\mathbf{k}(t), t]=-\left(\frac{d \bar{\theta}}{d z}\right) d Z_{3}[\mathbf{k}(t), t]
$$

where

$$
\theta^{\prime}(\mathbf{x}, t)=\int e^{i \mathbf{k}(t) \cdot \mathbf{x}} d \Theta[\mathbf{k}(t), t]
$$

Equations (24) and (25) constitute the governing RDT equations for homogeneous turbulent flow with nonneutral stratification $(d \bar{\theta} / d z)$ that is sheared $(d U / d z)$ 
with respect to the vertical $z$ direction. The rate of change of wavenumber due to mean shear becomes (Pope 2000)

$$
\frac{d k_{3}}{d t}=-k_{1} \frac{d U}{d z}
$$

The application of RDT to flows with both uniform mean wind shear and constant vertical mean potential temperature gradient has been accomplished previously, but such analyses had basically been limited to uniform shear with stable stratification (HH04; Segalini and Arnqvist 2015).

We employ the nondimensional time $\xi$ defined by (M94)

$$
\xi=\left(\frac{d U}{d z}\right) t
$$

and introduce $d \Theta[\mathbf{k}(t), t]$ in terms of the quantity $d Z_{4}[\mathbf{k}(t), t]:$

$$
d Z_{4}[\mathbf{k}(t), t]=\frac{g}{\bar{\theta}}\left(\frac{d U}{d z}\right)^{-1} d \Theta[\mathbf{k}(t), t] .
$$

The new quantity defined in (29) has been defined such that it has dimensions of $\mathrm{ms}^{-1}$, just like the velocity components $d Z_{i}$. From the above two transformations, the RDT equations including buoyancy in (24) and (25) can be rewritten as

$$
\frac{D}{D \xi} d Z_{l}[\mathbf{k}(\xi), \xi]=\mathbf{M}_{l m}[\mathbf{k}(\xi), \xi] d Z_{m}[\mathbf{k}(\xi), \xi]
$$

where

$$
\mathbf{M}_{l m}[\mathbf{k}(\xi), \xi]=\left[\begin{array}{cccc}
0 & 0 & \frac{2 k_{1}^{2}}{k^{2}}-1 & -\frac{k_{1} k_{3}}{k^{2}} \\
0 & 0 & \frac{2 k_{1} k_{2}}{k^{2}} & -\frac{k_{2} k_{3}}{k^{2}} \\
0 & 0 & \frac{2 k_{1} k_{3}}{k^{2}} & -\left(\frac{k_{3}^{2}}{k^{2}}-1\right) \\
0 & 0 & -\mathrm{Ri} & 0
\end{array}\right] .
$$

The Richardson number (Ri) is defined as (Kaimal and Finnigan 1994)

$$
\mathrm{Ri}=\frac{(g / \bar{\theta}) d \bar{\theta} / d z}{(d U / d z)^{2}}
$$

where $\bar{\theta}$ is the mean temperature representative of the height(s) of interest, with $d U / d z$ and $d \bar{\theta} / d z$ similarly representative.

\section{c. Initial conditions: Isotropic turbulence}

For a mean shear $d U / d z$ and from (27) and (28), the wavenumber vector after a dimensionless strain time $\xi$ is given as

$$
\mathbf{k}(\xi)=\left(k_{10}, k_{20}, k_{30}-k_{10} \xi\right)
$$

this gives the evolution of the wavenumber vector from an initial wavenumber vector $\mathbf{k}_{0}=\mathbf{k}(0)=\left(k_{10}, k_{20}, k_{30}\right)$.

\section{1) VELOCITY SPECTRA}

We assume a state of isotropic turbulence for the initial conditions. For the velocity components, the isotropic tensor is given as (Pope 2000)

$$
\Phi_{i j}\left(\mathbf{k}_{0}, 0\right)=\frac{E(k)}{4 \pi k^{2}}\left(\delta_{i j}-\frac{k_{i} k_{j}}{k^{2}}\right),
$$

which satisfies (conjugate) symmetry and the incompressibility conditions (8) and (9). We use the von Kármán (1948) form

$$
E(k)=\alpha \varepsilon^{2 / 3} L^{5 / 3} \frac{(k L)^{4}}{\left[1+(k L)^{2}\right]^{17 / 6}}
$$

for the isotropic energy spectrum $E(k)$, where $L$ is the length scale characteristic of energy-containing eddies, and $\alpha \approx 1.7$ is the (spectral) Kolmogorov constant. The single-point isotropic velocity spectra can be written analytically in streamwise-wavenumber space using (14), (34), and (35) as (Tennekes and Lumley 1972)

$$
F_{1}\left(k_{1}\right)=\frac{9}{55} \alpha \varepsilon^{2 / 3} \frac{1}{\left(L^{-2}+k_{1}^{2}\right)^{5 / 6}}
$$

and (Pope 2000)

$$
F_{2}\left(k_{1}\right)=F_{3}\left(k_{1}\right)=\frac{3}{110} \alpha \varepsilon^{2 / 3} \frac{3 L^{-2}+8 k_{1}^{2}}{\left(L^{-2}+k_{1}^{2}\right)^{11 / 6}} .
$$

\section{2) TeMPeRATURE SPECTRUM}

For temperature, the isotropic three-dimensional spectrum is given as

$$
\Phi_{\theta \theta}\left(\mathbf{k}_{0}, 0\right)=\frac{S(k)}{4 \pi k^{2}},
$$

where $S(k)$ is the potential energy spectrum containing the form of the inertial subrange (Kaimal and Finnigan 1994):

$$
S(k)=\beta_{1} \varepsilon^{-1 / 3} \varepsilon_{\theta} L^{5 / 3} \frac{(k L)^{2}}{\left[1+(k L)^{2}\right]^{11 / 6}} .
$$


Analogous to the TKE rate equation and scalar TKE spectrum, in (39), $\varepsilon_{\theta}$ is the rate of destruction of (half) the temperature variance, and $\beta_{1}=0.8$ is a universal constant (Kaimal et al. 1972). The initial length scale of the temperature spectrum is assumed to be identical to that of the velocity spectrum. The initial onedimensional temperature spectrum can be derived from (39) as

$$
F_{\theta}\left(k_{1}\right)=\frac{3}{10} \beta_{1} \varepsilon^{-1 / 3} \varepsilon_{\theta} \frac{1}{\left(L^{-2}+k_{1}^{2}\right)^{5 / 6}} .
$$

From (29), (38), and (39),

$$
\Phi_{44}\left(\mathbf{k}_{0}, 0\right)=\frac{S^{\prime}(k)}{4 \pi k^{2}},
$$

where

$$
S^{\prime}(k)=\beta \eta_{\theta} \frac{1+(k L)^{2}}{(k L)^{2}} E(k),
$$

$\beta=\beta_{1} / \alpha$, and

$$
\eta_{\theta}=\frac{\varepsilon_{\theta}}{\varepsilon}\left[\frac{g}{\bar{\theta}}\left(\frac{d U}{d z}\right)^{-1}\right]^{2}
$$

\section{d. Stationarity and eddy lifetime}

The spectral tensor $\boldsymbol{\Phi}(\mathbf{k}, \xi)$ is time dependent (via $\xi)$, and the eddies will stretch or compress over time, depending upon their orientation relative to the plane of uniform shear. But assuming that this distortion occurs for an infinitely long time is unrealistic; the eddies must break down at some point, and this breakdown should depend on the size of an eddy. To make the spectral tensor stationary-in effect removing the time dependenceand account for the size-dependent breakdown of eddies, we use the wavenumber-dependent eddy lifetime of M94:

$$
\tau(k)=\Gamma\left(\frac{d U}{d z}\right)^{-1}(k L)^{-2 / 3}\left\{{ }_{2} F_{1}\left[\frac{1}{3}, \frac{17}{6} ; \frac{4}{3} ; \frac{-1}{(k L)^{2}}\right]\right\}^{-1 / 2}
$$

where $\Gamma$ is a parameter to be determined, and ${ }_{2} F_{1}$ is the "ordinary" or "Gaussian" hypergeometric function. We make the spectral tensor stationary by replacing $t$ in (28) with the wavenumber-dependent eddy lifetime given in (44), and the anisotropic spectral tensor becomes (see the appendix)

$$
\begin{aligned}
& \boldsymbol{\Phi}\left(\mathbf{k}, \alpha \varepsilon^{2 / 3}, L, \Gamma, \mathrm{Ri}, \beta \eta_{\theta}\right) \\
& \quad=\alpha \varepsilon^{2 / 3} L^{11 / 3} \boldsymbol{\Phi}\left(\mathbf{k} L, 1,1, \Gamma, \mathrm{Ri}, \beta \eta_{\theta}\right) .
\end{aligned}
$$

The derived spectral tensor, including the effect of uniform shear and buoyancy (via uniform lapse rate), contains five parameters to be determined:

- $\alpha \varepsilon^{2 / 3}$, where $\varepsilon$ is the (TKE) dissipation rate and $\alpha$ is the spectral Kolmogorov constant;

- $L$, which represents the size of the energy-containing eddies;

- $\Gamma$, a measure of turbulence anisotropy;

- $\mathrm{Ri}$, characterizing atmospheric stability;

- $\eta_{\theta}$, normalized rate of destruction of temperature variance [see (43)].

The five parameters can be determined from observed single-point spectra and cospectra and are used as inputs to estimate the model cross-spectra defined in (14). Again, we are neglecting Earth's rotation, so for a given $d U / d z$ and $d \bar{\theta} / d z$, the four-dimensional spectral tensor $\boldsymbol{\Phi}$ in (45) should satisfy left-right symmetry; thus the modeled $\left\langle u^{\prime} v^{\prime}\right\rangle=\left\langle v^{\prime} w^{\prime}\right\rangle=0$ [i.e., the second element in the symmetric group given by (2.14) of M94].

Including buoyancy, the roles of the model parameters $\alpha \varepsilon^{2 / 3}, L$, and $\Gamma$ in maintaining variances and covariances are similar to that described in Mann (1994a, 1998, 2000) and Chougule et al. (2015). The model parameter Ri determines the sign of the temperature fluxes and affects the location and amplitude of spectral peaks. For stable cases, an increase in Ri (holding other parameters constant) results in shifting the peaks of velocity spectra to smaller scales and reducing the peak spectral amplitudes (including amplitude of the peak of $u w$ ), with the $u$ spectra much more influenced than the $v$ and $w$ spectra. The peak amplitudes of the $\theta$ spectrum and $u \theta$ cospectrum increase, with the amplitude of (negative) $w \theta$ increasing, for increasingly stable $(\mathrm{Ri}>0)$ conditions. The effects of Ri on stable spectra and cospectra are shown in Fig. 1. In stable cases, the effect of an increase in $\eta_{\theta}$ is opposite to that of $\mathrm{Ri}$, except that the peak magnitude of the $\theta$ spectrum increases (similar to an increase in $\mathrm{Ri}$ ), with the peak of the temperature autospectrum shifting to higher wavenumbers. In unstable cases, an increase in the magnitude of $\mathrm{Ri}$ has a similar effect on the $\theta$ spectra and $u \theta$ and $w \theta$ cospectra as that in the stable case, whereas the peak magnitudes of velocity spectra increase; the $u w$ cospectrum peak shifts downward toward lower wavenumbers (as illustrated in Fig. 2), while $\eta_{\theta}$ affects the spectra in a manner similar to Ri.

\section{Observations and method}

We validate our model using the observations from the HATS field program, which was carried out in September 2000 near Kettleman City, California (Horst et al. 2004). 

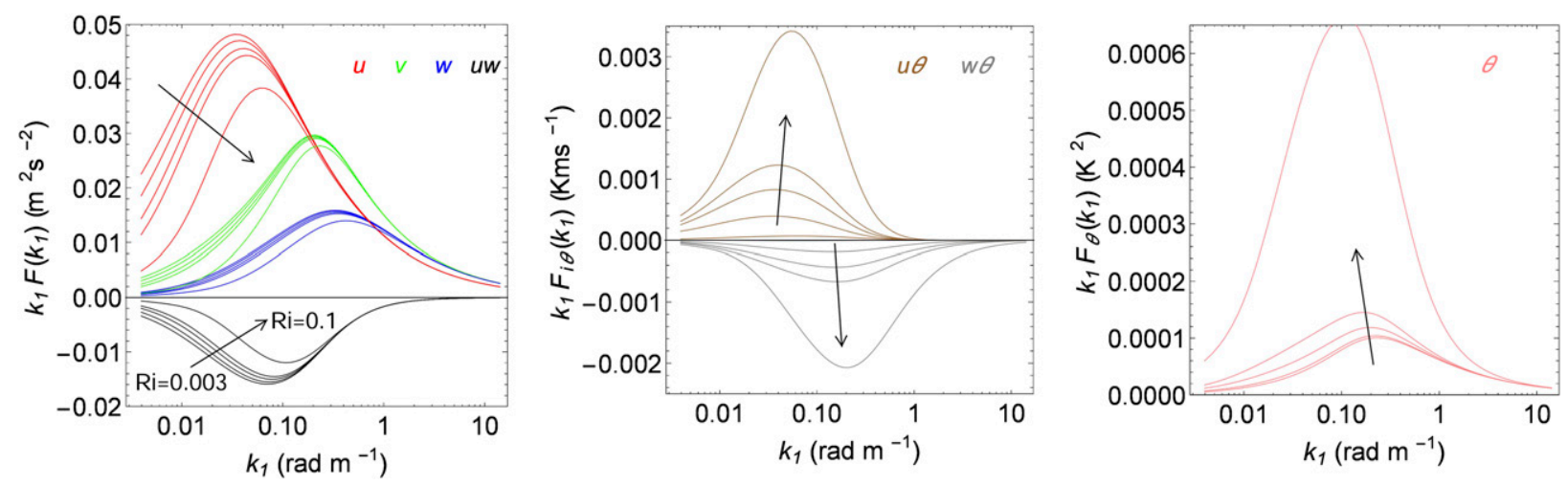

FIG. 1. Effect of Richardson number (Ri) on model spectra and cospectra for stable cases $(\mathrm{Ri}>0)$. In the direction of the arrow, $\mathrm{Ri}=0.003,0.01,0.02,0.03$, and 0.1 .

\section{a. Experiment}

A number of different setup configurations are deployed in the HATS experiment, where two horizontal arrays of sonic anemometers, each measuring temperature and three-dimensional wind velocity with a sampling rate of $20 \mathrm{~Hz}$, are placed at different heights from the ground. As shown in Fig. 3, a horizontal $(s$ array) of five sonic anemometers are placed at one height $z_{s}$ from the ground, and nine sonic anemometers ( $d$ array) are mounted at a second height $z_{d}$ parallel to and with a common center and endpoints as the s-array. The sonic anemometers in the horizontal $s$ and $d$ arrays are separated by spacings of $S_{s}$ and $S_{d}$, respectively. More information about the HATS experiment can be found in Horst et al. (2004) and Sullivan et al. (2003).

\section{b. Method}

We estimate the component velocity autospectra and cospectra from the measured 30-min time series via

$$
F_{i j}(f, z) \equiv\left\langle\hat{u}_{i}(f) \hat{u}_{j}^{*}(f)\right\rangle
$$

and the temperature spectrum and the componentwise kinematic heat fluxes are calculated as

$$
\begin{aligned}
& F_{\theta}(f, z) \equiv\left\langle\hat{\theta}(f) \hat{\theta}^{*}(f)\right\rangle \quad \text { and } \\
& F_{i \theta}(f, z) \equiv\left\langle\hat{u}_{i}(f) \hat{\theta}^{*}(f)\right\rangle \quad \text { for } \quad i=\{1,3\},
\end{aligned}
$$

where $\hat{u}_{i}(f)$ and $\hat{\theta}(f)$ are the complex-valued Fourier transform of the $i$ th velocity component and potential temperature, respectively, measured at height $z$.

The atmospheric stability is quantified in terms of the ratio $z / L_{o}$, where the Obukhov length $L_{o}$ is defined as (Kaimal and Finnigan 1994)

$$
L_{o}=\frac{-u_{\star}^{3}}{\kappa\left(g / \bar{\theta}_{0}\right)\left\langle w^{\prime} \theta^{\prime}\right\rangle_{0}}
$$
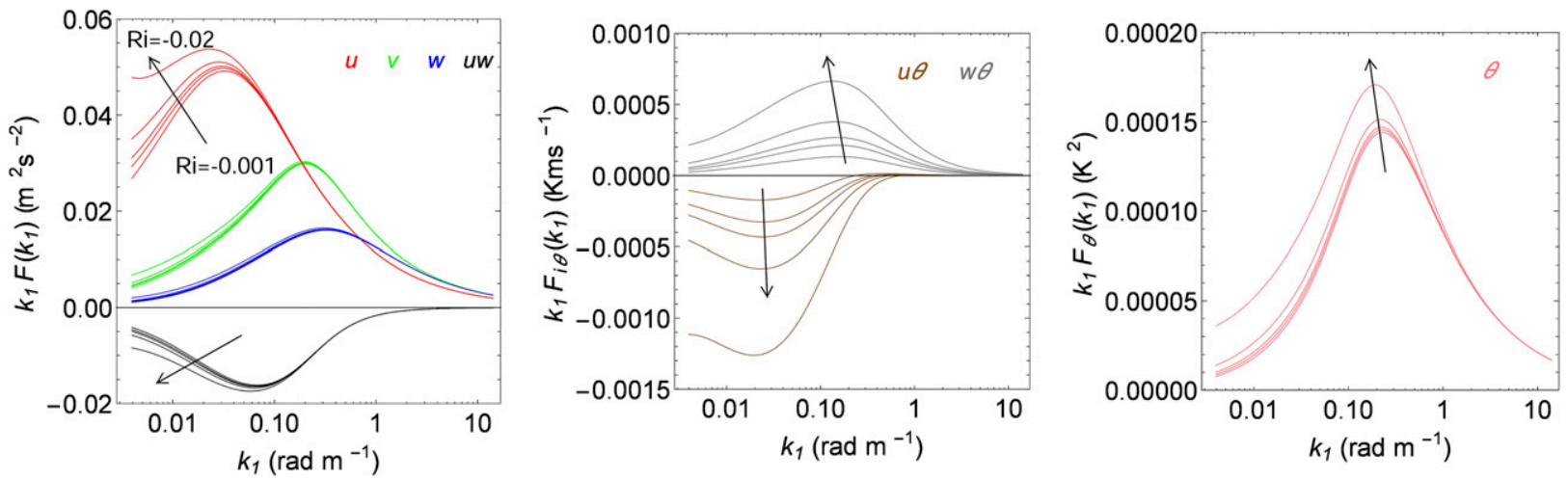

FIG. 2. Ri influencing unstable model spectra and cospectra $(\mathrm{Ri}<0)$. In the direction of the arrow, $\mathrm{Ri}=-0.001,-0.004,-0.006,-0.01$, and -0.02 . 


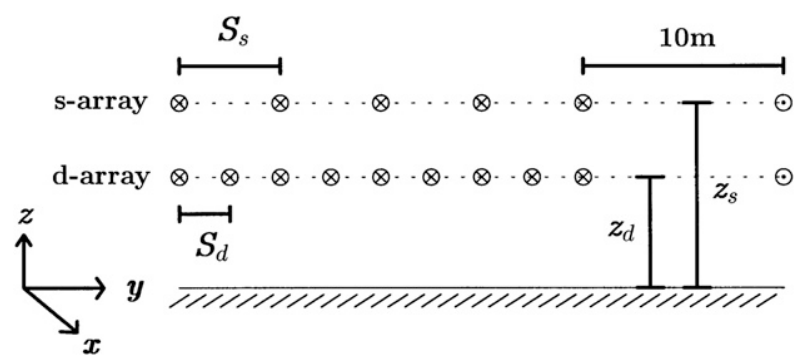

FIG. 3. The HATS experimental setup: the sonic anemometers $(\otimes)$ are mounted in the single and double arrays at $\left(z_{s}, z_{d}\right)$ above the ground, respectively. From Fig. 1 of Horst et al. (2004). Copyright American Meteorological Society. Used with permission. where $u_{\star}$ is the surface friction velocity, $\kappa=0.4$ is the von Kármán constant, $\bar{\theta}_{0}$ is the mean surface-layer temperature, and $\left\langle w^{\prime} \theta^{\prime}\right\rangle_{0}$ is the vertical flux of potential temperature in the surface layer.

We adopt an automatic fit procedure by performing $\chi^{2}$ fits (M94) of the model to the measured power spectra in (46)-(48) using Taylor's hypothesis $\left(k_{1}=2 \pi f / U\right)$ to obtain the five model parameters for each 30-min case. The $\chi^{2}$ fit minimizes the sum of squared differences between the theoretical and the estimated spectra and cospectra, and the $\chi^{2}$ equation of M94 is modified by including $\theta$ spectrum and cospectra $u \theta$ and $w \theta$; it is given as

$$
\begin{aligned}
\chi^{2}\left(\alpha \varepsilon^{2 / 3}, L, \Gamma, \mathrm{Ri}, \eta_{\theta}\right)= & \frac{1}{\left|F_{13}^{\max }\right|} \sum_{n=1}^{N}\left[F_{13, T}\left(k_{n}\right)-F_{13}\left(k_{n}\right)\right]^{2}+\sum_{l=1}^{4} \frac{1}{\left|F_{l}^{\max }\right|} \sum_{n=1}^{N}\left[F_{l, T}\left(k_{n}\right)-F_{l}\left(k_{n}\right)\right]^{2} \\
& +\frac{1}{\left|F_{14}^{\max }\right|} \sum_{n=1}^{N}\left[F_{14, T}\left(k_{n}\right)-F_{14}\left(k_{n}\right)\right]^{2}+\frac{1}{\left|F_{34}^{\max }\right|} \sum_{n=1}^{N}\left[F_{34, T}\left(k_{n}\right)-F_{34}\left(k_{n}\right)\right]^{2},
\end{aligned}
$$

where $N$ is the number of wavenumbers in the estimated spectra, subscript $T$ corresponds to the model spectra and cospectra, and $F^{\mathrm{max}}$ is the corresponding maximum value in the measured spectra and cospectra. Also, additionally, we develop the fit routine such that it is possible, for practical purposes, to constrain fits by filtering some of both the low- and high-wavenumber fluctuations (shown by dotted lines in the spectral fit Figs. 4-8), corresponding, respectively, to mesoscale and the noise in the spectra that might occur. As mentioned earlier, the fourth component is the temperature premultiplied by a factor of $(g / \bar{\theta}) /(d U / d z)$, as given in (29).

\section{Results and discussion}

Just as the Fourier temperature component $d \Theta$ has been redimensionalized by the prefactor $(g / \bar{\theta})(d U / d z)^{-1}$ to become $d Z_{4}$ (to have the same dimensions as the Fourier velocity components $d Z_{i}$ ), the model temperature spectrum and the corresponding cospectra become

$$
\begin{aligned}
& F_{\theta}\left(k_{1}\right)=\left[\frac{g}{\bar{\theta}}\left(\frac{d U}{d z}\right)^{-1}\right]^{-2} F_{4}\left(k_{1}\right) \text { and } \\
& F_{i \theta}\left(k_{1}\right)=\left[\frac{g}{\bar{\theta}}\left(\frac{d U}{d z}\right)^{-1}\right]^{-1} F_{i 4}\left(k_{1}\right) \text { for } i=\{1,3\} .
\end{aligned}
$$

\section{a. Spectra}

The results from the model power spectra and cospectra are compared with the observations for two cases: case I for stable stratification (positive $z / L_{o}$ ) and case II for unstable stratification $\left(z / L_{o}<0\right)$. Case I consists of four $z / L_{o}$ values varying from near-neutral stable $\left(z / L_{o}=0.015\right)$ to highly stable $\left(z / L_{o}=0.13\right)$ conditions at $z=6 \mathrm{~m}$. Figures 4 and 5 show the measured and model-fitted autospectra and cospectra of velocity and temperature for case I. The corresponding $z / L_{o}$ values and the model parameters from the $\chi^{2}$ fits are shown in Table 1.

The model length scale $L$ and $\Gamma$ parameter are reduced with increasing $z / L_{o}$ in case I. The $\Gamma$ parameter is proportional to eddy lifetime, meaning that the turbulent eddies effectively live slightly longer in near-neutral stable conditions than in more stable stratification. Both the $\mathrm{Ri}$ and $\eta_{\theta}$ parameters increase with $z / L_{o}$. It is observed both from the model and the data that the velocity spectra and $u w$ cospectrum are reduced with increased $z / L_{o}$, as indicated in Fig. 4, whereas the temperature spectrum and the temperature fluxes are increased. However, in Fig. 5 we notice that the measured streamwise temperature flux is reduced with increased stratification, which may be due to the low mean wind speed and small (or damped) variability in $u$. This reduction in the streamwise temperature flux is also reproduced by the model via $\chi^{2}$ fits (as shown in Fig. 5), where $\alpha \varepsilon^{2 / 3}$ is reduced, and $\eta_{\theta}$ is only slightly increased from $z / L_{o}=0.095$ to 0.13 (this increment in $\eta_{\theta}$ is small relative to the large increment in $\eta_{\theta}$ from $z / L_{o}=0.015$ to 0.05 for the fits in Fig. 4). The streamwise temperature flux is observed to be larger than the vertical temperature flux, and the ratio $\langle u \theta\rangle /\langle w \theta\rangle$ reduces with the 

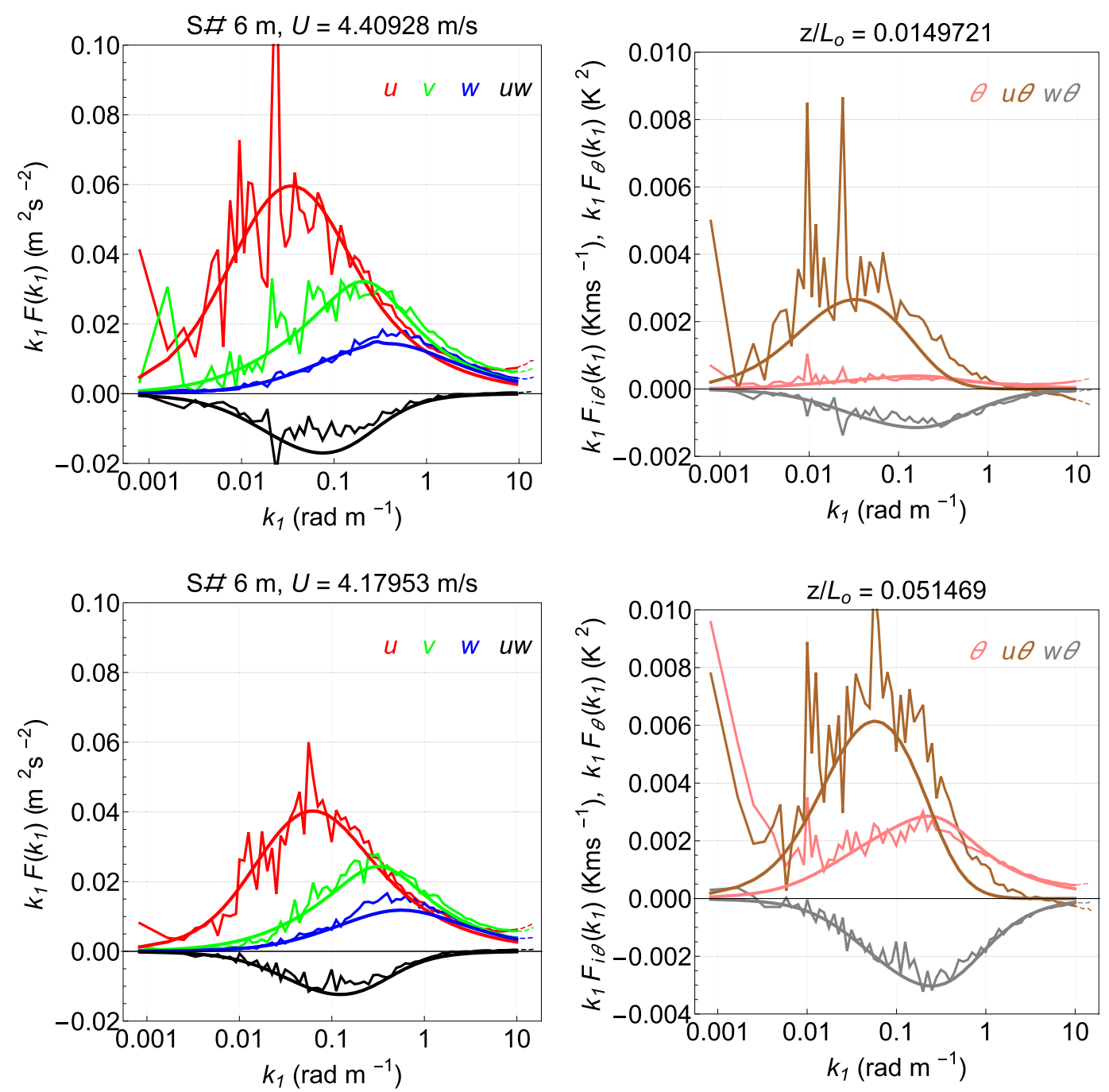

FIG. 4. Model spectra and cospectra fitted with observations via $\chi^{2}$-fit method at $z=6 \mathrm{~m}$ for (top) $z / L_{o}=0.015$ and (bottom) $z / L_{o}=0.05$. Smooth lines are model predictions, and ragged lines are the observations.

stratification. This is due to the combined effect of reduced length scale $L$, and the $\Gamma$ parameter, which is also decreased, implying smaller and smaller-scale separation between $u$ and $w$. For stable conditions described by $z / L_{o}=0.015$ and $0.05\left(z / L_{o}=0.095\right.$ and 0.13$)$, observed ratios $\langle u \theta\rangle /\langle w \theta\rangle$ are roughly 3.5 and 3.3 (3.0 and 2.3); the corresponding ratios from the model fits are 2.0 and 1.8 (1.7 and 1.5). Although the model predicts the $\theta$ spectrum and $w \theta$ cospectrum reasonably well, there is a systematic underestimation of the $u \theta$ cospectrum by the model, as seen from Figs. 4 and 5, and therefore the ratios $\langle u \theta\rangle /\langle w \theta\rangle$ from the model are smaller than observed. The model is able to reproduce the decrease in $\langle u \theta\rangle /\langle w \theta\rangle$ values with the stratification, however.

The model spectra and cospectra fitted to measurements at different heights for the unstable stratification case II are shown in Figs. 6-8. The corresponding $z / L_{o}$, along with fit model parameters, are shown in Table 2 . The turbulence close to the ground is mostly driven by surface friction so that the dissipation rate is higher close to the ground, which can be seen by looking at the $\alpha \varepsilon^{2 / 3}$ values in Table 2. There is also a sharp increase in the streamwise energy content at lower wavenumbers in unstable cases, which also causes an increase in the energy content of the $u w$ and $u \theta$ cospectra at lower frequencies. Therefore, we only used measured spectral content from $k_{1} \geq 0.01$ in the spectral fits for unstable regimes to obtain the five model parameters for case II. The length scale $L$ increases with height, as expected. The $\Gamma$ values from the spectral fits are noted to be relatively smaller in the unstable case. Also, in case II, a common notation from Table 2 can be that the $\Gamma$ parameter decreases slightly, whereas $\eta_{\theta}$ increases with stronger stratification, which is also consistent with the 

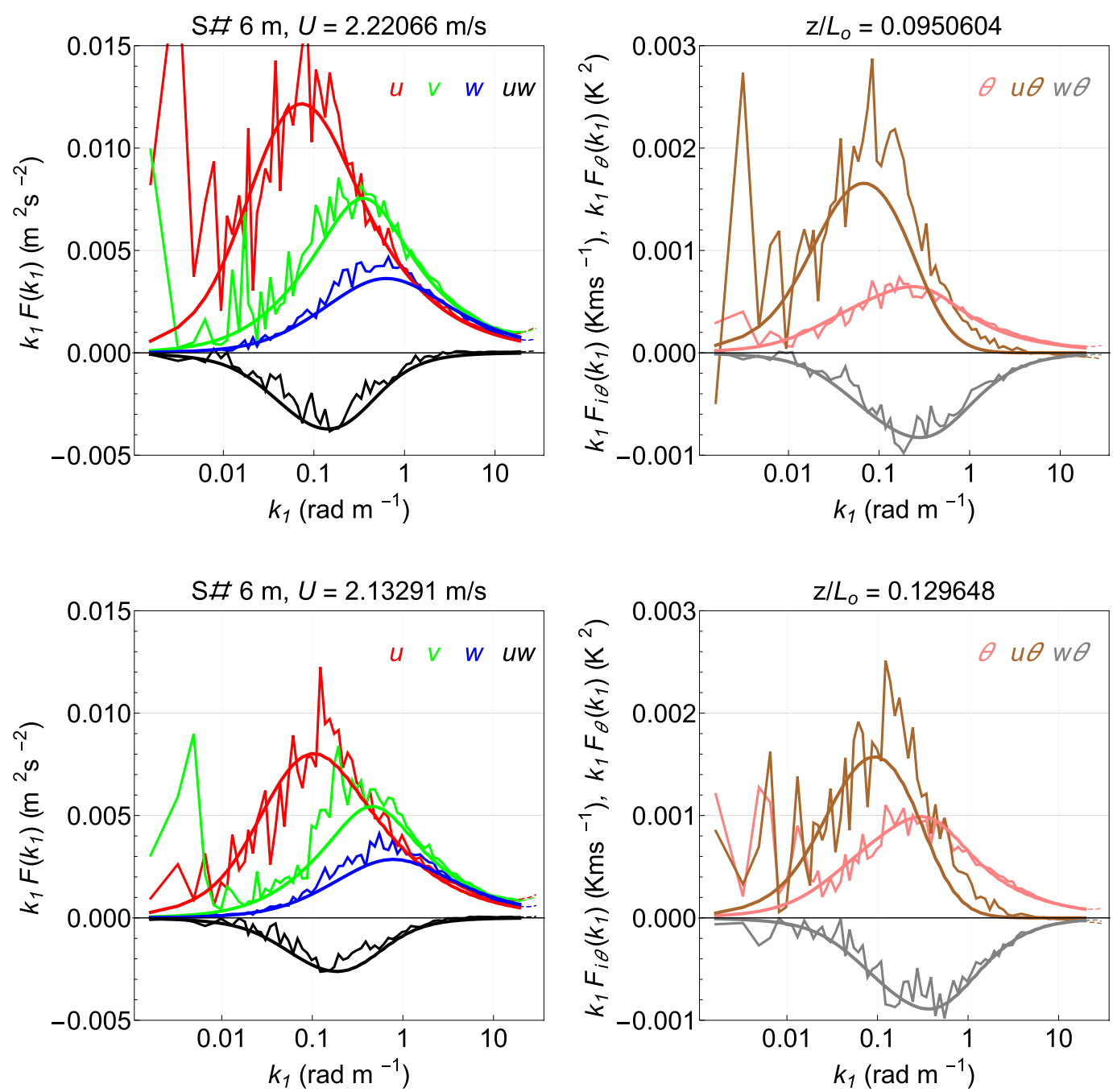

FIG. 5. Model spectra and cospectra compared with the spectra observed at $z=6 \mathrm{~m}$ for stable conditions [(top) $z / L_{o}=0.095$ and (bottom) $\left.z / L_{o}=0.13\right]$. Notation follows Fig. 4.

$\Gamma$ and $\eta_{\theta}$ values in Table 1 for case I. A slight increase in $\Gamma$ is observed for conditions approaching neutral, both from stable and unstable regimes.

Buoyancy affects turbulence, and that is expressed more so by a change in length scale, which is also noted in Chougule et al. (2015). The dissipation rate $\varepsilon$ depends primarily upon mean wind speed, which is not explicitly included in the model. In the stable case I, for example, $\alpha \varepsilon^{2 / 3}$ has the same value for $z / L_{o}=0.015$ and 0.05 , but $L$ is reduced for $z / L_{o}=0.05$. At $z=6 \mathrm{~m}, \alpha \varepsilon^{2 / 3}$ is larger for $z / L_{o}=0.05$ than for $z / L_{o}=-0.03$, while $L$ is smaller. The effect of buoyancy on the relative level of inertial-range variance in temperature (i.e., the right graphs in Figs. 4-8) is expressed in terms of $\eta_{\theta}$, which increases with stability magnitude $\left|z / L_{o}\right|$. The spectral peaks corresponding to length scales of the $\theta$ spectrum and $w \theta$ cospectrum coincide with each other (with slight differences in unstable cases), and the peak scales for $u \theta$ are larger than those for $\theta$ and $w \theta$ (which is observed in both stable and unstable conditions); the model reproduces the same. The model also captures the switch in signs of $u \theta$ and $w \theta$ occurring in stable versus unstable cases, as driven by the fits.

The velocity and temperature spectra follow Kolmogorov's power law in the inertial subrange [i.e., $F\left(k_{1}\right) \propto k_{1}^{-5 / 3}$ ],

TABLE 1. The spectral tensor parameters determined from $\chi^{2}$ fits for case I.

\begin{tabular}{cccccc}
\hline \hline & \multicolumn{5}{c}{ Model parameters } \\
\cline { 2 - 6 }$z / L_{o}$ & $\alpha \varepsilon^{2 / 3}\left(\mathrm{~m}^{4 / 3} \mathrm{~s}^{-2}\right)$ & $L(\mathrm{~m})$ & $\Gamma$ & $\mathrm{Ri}$ & $\eta_{\theta}$ \\
\hline 0.015 & 0.074 & 5.66 & 4.20 & 0.007 & 0.0004 \\
0.050 & 0.074 & 3.93 & 3.87 & 0.022 & 0.0025 \\
0.095 & 0.025 & 3.54 & 3.82 & 0.034 & 0.0053 \\
0.130 & 0.022 & 2.85 & 3.46 & 0.048 & 0.0096 \\
\hline
\end{tabular}



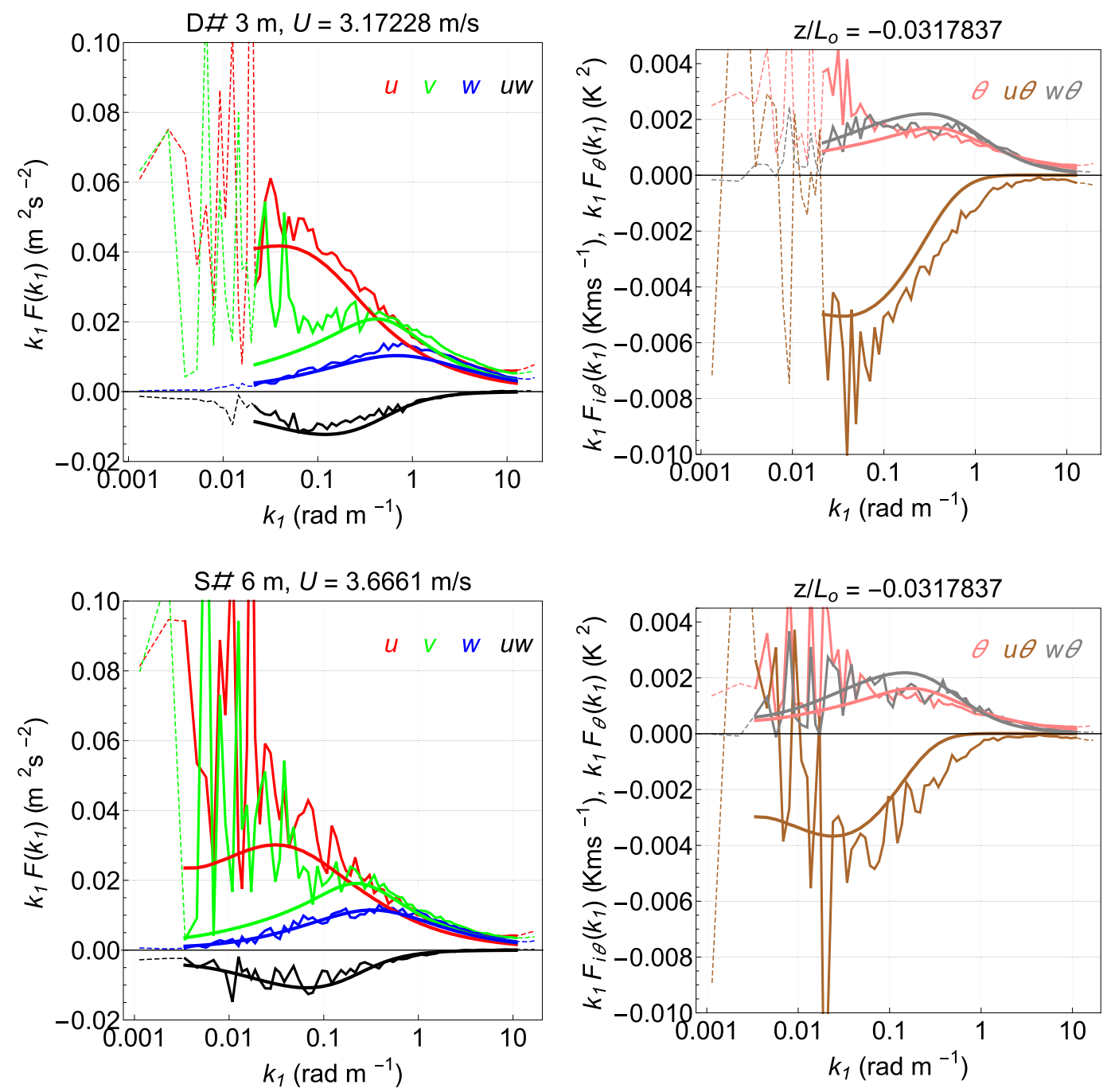

FIG. 6. The modeled (slightly) unstable spectra and cospectra fitted with observations at (top) $z=3$ and (bottom) $z=6 \mathrm{~m}$ for $z / L_{o}=-0.03$. Smooth lines are model predictions, and ragged lines are the observations.

whereas the $u w$ and $w \theta$ cospectra are proportional to $k_{1}^{-7 / 3}$ in the inertial subrange, shown by the data and also predicted by the model. Our model predicts a powerlaw exponent closer to -3 for the $u \theta$ cospectrum under stable and unstable stratification. The data from stable stratifications shows spectral (log-log) slopes closer to -3 in the $u \theta$ cospectra. In contrast to the situation for the $\theta$ spectrum and $w \theta$ cospectrum, the unstable $u \theta$ inertialrange cospectrum does not agree with our model predictions, with observed data indicating a spectral slope of $-7 / 3$. The $u \theta$ slopes are shown in $\log -\log$ plots in Fig. 9 from both the model and the data for stable and unstable cases. In contrast, Kaimal et al. (1972) noted an averaged slope of $-5 / 2$ for stable cases. Wyngaard and Coté (1972) predicted a slope of -3 for the inertial-range $u \theta$ cospectrum, while slopes from their data imply values closer to $-7 / 3$, albeit at a higher height of $22.6 \mathrm{~m}$.
The velocity and temperature spectra and corresponding cospectra were initially estimated from time series at each sonic anemometer in the $s$ and $d$ arrays, and averaged over the sonic anemometers in each respective ( $s$ or $d$ ) row:

$$
F_{l m}(f, z) \equiv \frac{1}{P} \sum_{n=1}^{P}\left\langle\hat{\Im}_{l}(f) \hat{\Im}_{m}^{*}(f)\right\rangle_{n}
$$

where $P$ is the number of sonic anemometers in either the $s$ or $d$ array. For the comparisons of the model parameters in case II, we take $P=5$ and consider/use only the sonic anemometers in the $d$ array that are directly above/below those in the $s$ array.

Following M94 and HH04, Segalini and Arnqvist (2015) proposed a similar analytic model, but with different parameters describing the spectral tensor. We include temperature spectra, which are responsible for 

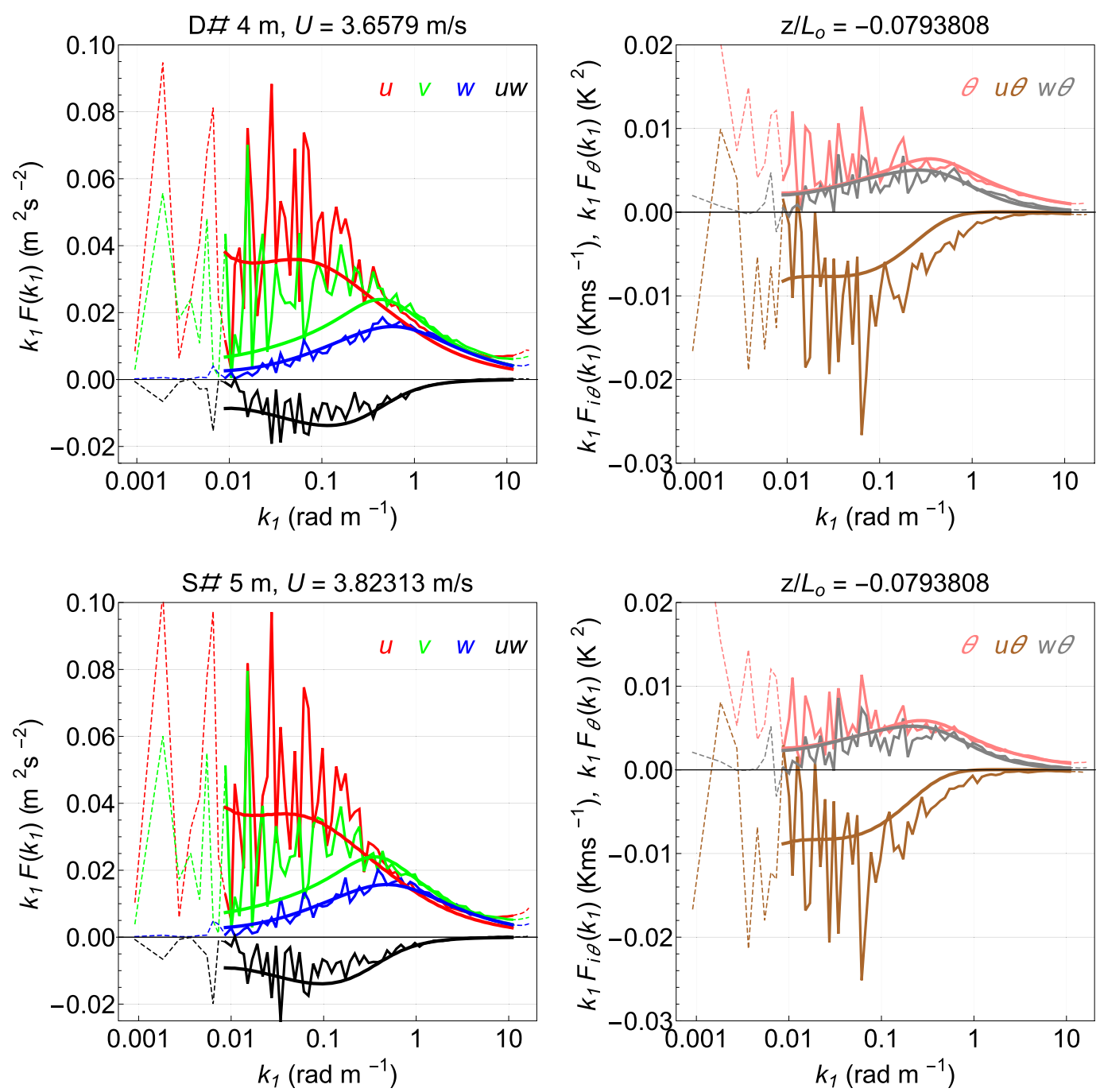

FIG. 7. Model spectra and associated cospectra are fitted with observations at (top) $z=4$ and (bottom) $z=5 \mathrm{~m}$ for $z / L_{o}=-0.08$. For notations, see Fig. 6 .

the destruction rate of the temperature variance; this is not included in Segalini and Arnqvist (2015). The predictions of $u w, u \theta$, and $w \theta$ cospectra in Segalini and Arnqvist (2015) show nonphysical kinks, whereas our model does not show such kinks. Two different length scales, one representing velocity and the other representing the scalar part, were assumed initially in Segalini and Arnqvist (2015). We take the single length scale both for the dynamic as well as the kinematic part, following Chougule (2013), and all the provided temperature spectra and associated fluxes are described by the same length scale. A stability-dependent eddy lifetime model is proposed in Segalini and Arnqvist (2015), resulting in an extra parameter in addition to the $\Gamma$ parameter. With the wavenumber-dependent eddy lifetime of M94, our model prediction is in reasonable agreement with observations under stable stratification. The $\Gamma$ values used in Segalini and Arnqvist (2015, their $K$ ) were systematically higher than the values reported previously from M94, contrary to their definition of eddy lifetime, which reduces the value of $\Gamma$ in stable conditions. The $\Gamma$ values from our model fits are also larger than those of M94 for stable stratification, however. As discussed earlier in the role of the model parameters, an increase in Ri in the stable case influences the $u$ spectra more in shifting its peak (where increasing Ri pushes the peak to higher wavenumbers), so in order to compensate for the effect of $u$ spectra being more affected, the $\Gamma$ parameter must be increased. Further, the increase of $\Gamma$ with increasing $\mathrm{Ri}$ is also consistent with the physical observation that shear increases with an increasing degree of stable conditions, which then in turn is consistent with (44), showing that $\Gamma$ increases with increasing $d U / d z$ for 

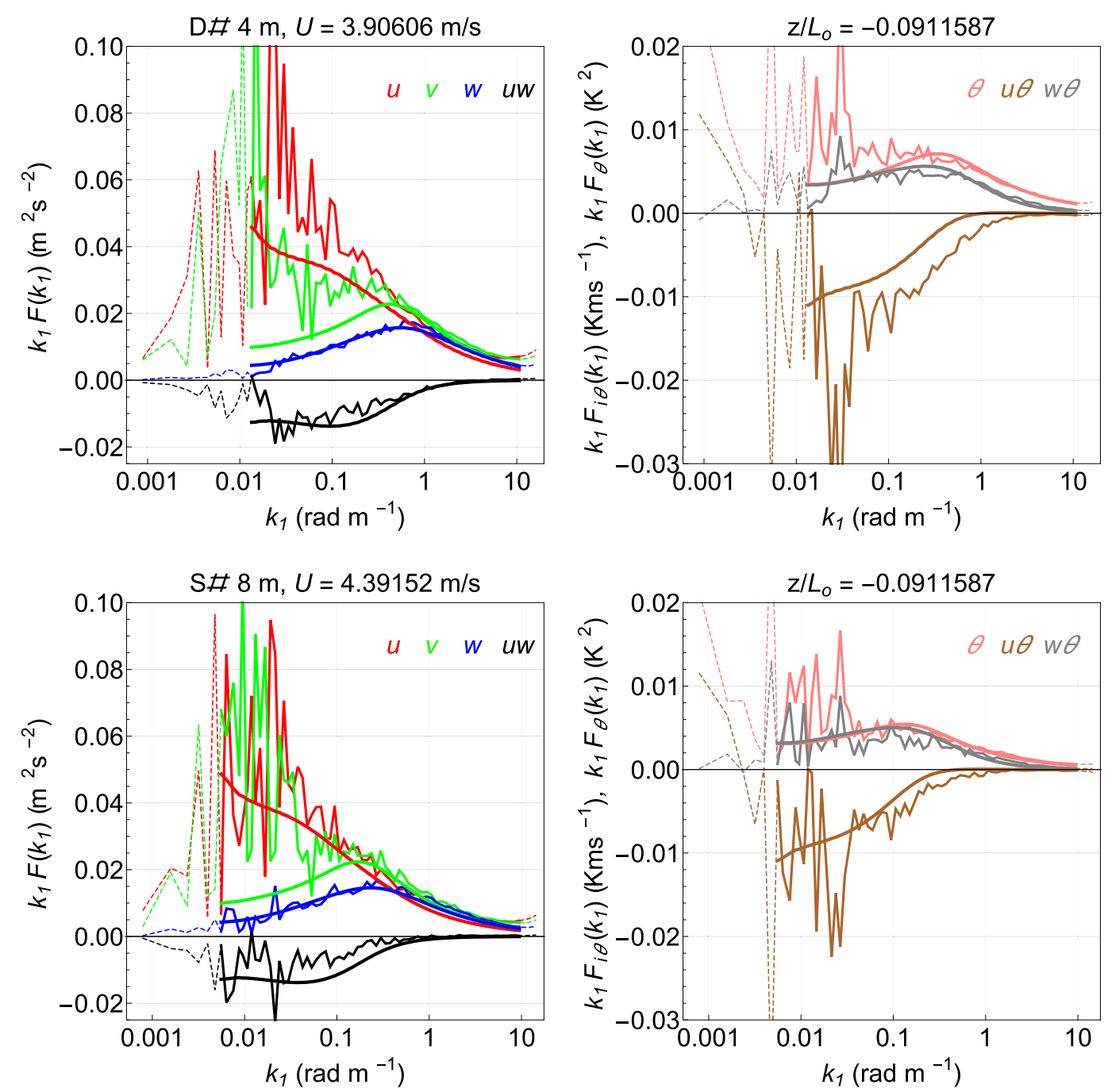

FIG. 8. Model spectral fits with the observations for $z / L_{o}=-0.09$ at (top) $z=4$ and (bottom) $z=8 \mathrm{~m}$. Notations are similar to previous figures.

fixed eddy lifetime (i.e., for fixed $k$ ). We further note that our model underestimates the $u \theta$ cospectrum, and one might argue that it might be due to limitations in the M94 lifetime model; if we exclude the temperature spectrum from the $\chi^{2}$-fit procedure, we get better agreement with the $u \theta$ cospectrum for stable stratification. However, while one might argue to include $F_{\theta \theta}$ in our results (as mentioned earlier), this is a practical choice, because of noise often contaminating $F_{\theta \theta}$, even into the inertial range.

We also include RDT predictions for unstable stratification, which was not included in Segalini and Arnqvist (2015). Additionally, as discussed in the next subsection, we also show coherence predictions for lateral separation, including the temperature coherence.

\section{b. Cross-spectra}

Winds within $\pm 13^{\circ}$ of normal to the plane of the sonic $s$ and $d$ arrays are selected, and the cross-spectra are rotated according to (20). The model coherences and

TABLE 2. Five spectral tensor parameters obtained from $\chi^{2}$ fits for case II.

\begin{tabular}{ccccccc}
\hline & & \multicolumn{5}{c}{ Model parameters } \\
\cline { 3 - 7 }$z / L_{o}$ & $z(\mathrm{~m})$ & $\alpha \varepsilon^{2 / 3}\left(\mathrm{~m}^{4 / 3} \mathrm{~s}^{-2}\right)$ & $L(\mathrm{~m})$ & $\Gamma$ & $\mathrm{Ri}$ & $\eta_{\theta}$ \\
\hline-0.03 & 3 & 0.080 & 2.74 & 3.80 & -0.022 & 0.005 \\
& 6 & 0.048 & 5.65 & 3.13 & -0.023 & 0.005 \\
-0.08 & 4 & 0.096 & 2.74 & 3.06 & -0.041 & 0.015 \\
& 5 & 0.084 & 3.56 & 2.86 & -0.041 & 0.013 \\
-0.09 & 4 & 0.089 & 2.97 & 2.66 & -0.057 & 0.035 \\
& 8 & 0.048 & 7.15 & 2.93 & -0.054 & 0.025 \\
\hline
\end{tabular}



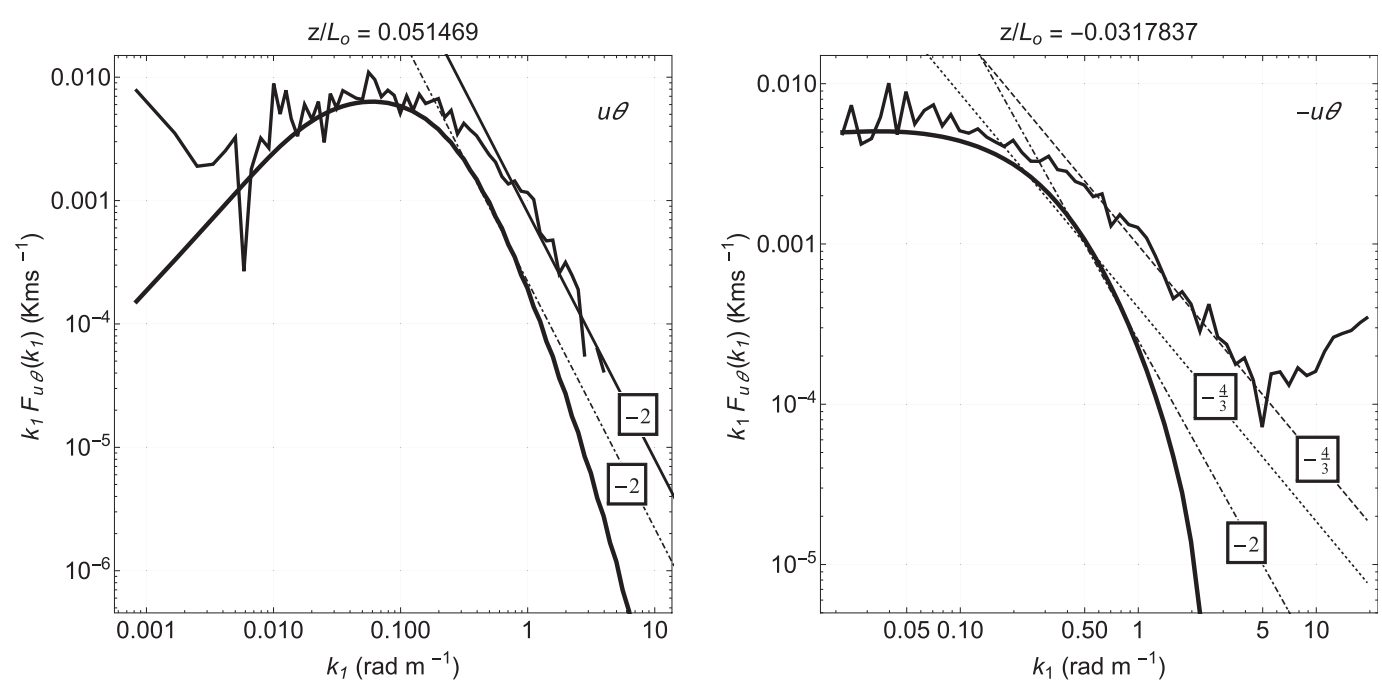

FIG. 9. On the $u \theta$ slopes $k_{1} F\left(k_{1}\right)$ vs $k_{1}$ : The model $u \theta$ cospectra (smooth curves) shown along with the data (ragged curves) in $\log -\log$ scale. Lines are slopes with the given values in framed insets for (left) stable and (right) unstable cases.

cross-spectral phases are calculated using (15) and (16), respectively. We show the results for two configurations for both positive and negative $z / L_{o}$. In one configuration, $s$ and $d$ arrays are placed at 5 and $4 \mathrm{~m}$, respectively, where we calculate coherences for vertical $(\Delta z=1 \mathrm{~m})$ and horizontal ( $\Delta y=0.63 \mathrm{~m}$ in the $s$ array) separations; these are shown in Figs. 10 and 11, respectively, both for stable $\left(z / L_{o}=0.08\right)$ and unstable $\left(z / L_{o}=-0.08\right)$ stratification. For the second configuration, we compare measured and model coherences between $s$ and $d$ arrays placed at 8 and $4 \mathrm{~m}$ for the stratifications $z / L_{o}=0.014$ and -0.09 , with the separations $\Delta z=4 \mathrm{~m}$ (Fig. 12) and $\Delta y=4.33$ and $8.66 \mathrm{~m}$ (Fig. 13 for $z / L_{o}=0.014$, Fig. 14 for $\left.z / L_{o}=-0.09\right)$. Both modeled and observed phases in the cross-spectra are shown in Figs. 15 and 16 for the vertical separations in the two configurations.

For vertical separations, the model spectra are fitted to the 30-min time series from the middle sonic anemometer of each $(s$ and $d$ ) array to obtain the model parameters, and the coherences from the measurements are calculated between the middle sonic anemometers. For smoother phases, however, we average the cross-spectra calculated from the data over the sonic anemometers according to (53). There was no significant difference in the model parameter values obtained from single sonic anemometers and those obtained from fitting averaged spectra.

The model performs relatively better for the lateral than the vertical separations. With larger vertical separation, the model coherence departs from observed, with overestimations of the coherence as seen in Fig. 12. The reason behind this can be that the homogeneity assumption breaks down in the vertical direction, as seen in (7) and (33), where $d U / d z$ close to the ground might have changed (too) dramatically over the heights considered. The turbulent eddies at low wavenumbers (or larger size) are more affected because of the shear and presence of the ground than the smaller-scale (more isotropic) eddies. For lateral separations, however, the departures from the measurements are relatively small. Both the model and measured coherences decrease with separation distance.

As discussed in section $2 \mathrm{~d}$, the spectral velocity tensor has left-right symmetry. The symmetry is tested with the measurements by calculating the coherence between the sonics in the second configuration for $\Delta z=4 \mathrm{~m}$ and $\pm \Delta y=2.17 \mathrm{~m}$, as illustrated in Fig. 17. The model coherences are compared for the above description in Fig. 18 for stable and unstable conditions. The model coherences shown by smooth lines for $\pm \Delta y$ coincide with each other, whereas the observed coherences shown by the ragged lines also indicate left-right symmetry.

Atmospheric stability affects the coherence, which increases from stable to unstable stratification. Further, the (thermal) stability has the most noticeable effect on the $w$ coherence, while the $u$ coherence is less affected by it, which was noted in Chougule et al. (2015) for vertical separations. For $\Delta z=1 \mathrm{~m}$ as in Fig. 10, the $w$ and $v$ coherences are slightly larger in unstable cases. For $\Delta z=4 \mathrm{~m}$, the measured $w$ and $v$ coherences are also larger for negative $z / L_{o}$, as seen in Fig. 12. For the model coherence, however, the effect of stability is less profound because of the fact that the model overestimates coherence more in stable than in unstable stratification. For a lateral separation of $\Delta y=0.63 \mathrm{~m}$, both the model and observed $w$ coherences are slightly larger for unstable $\left(z / L_{o}<0\right)$ regimes, as can be seen from Fig. 11. 


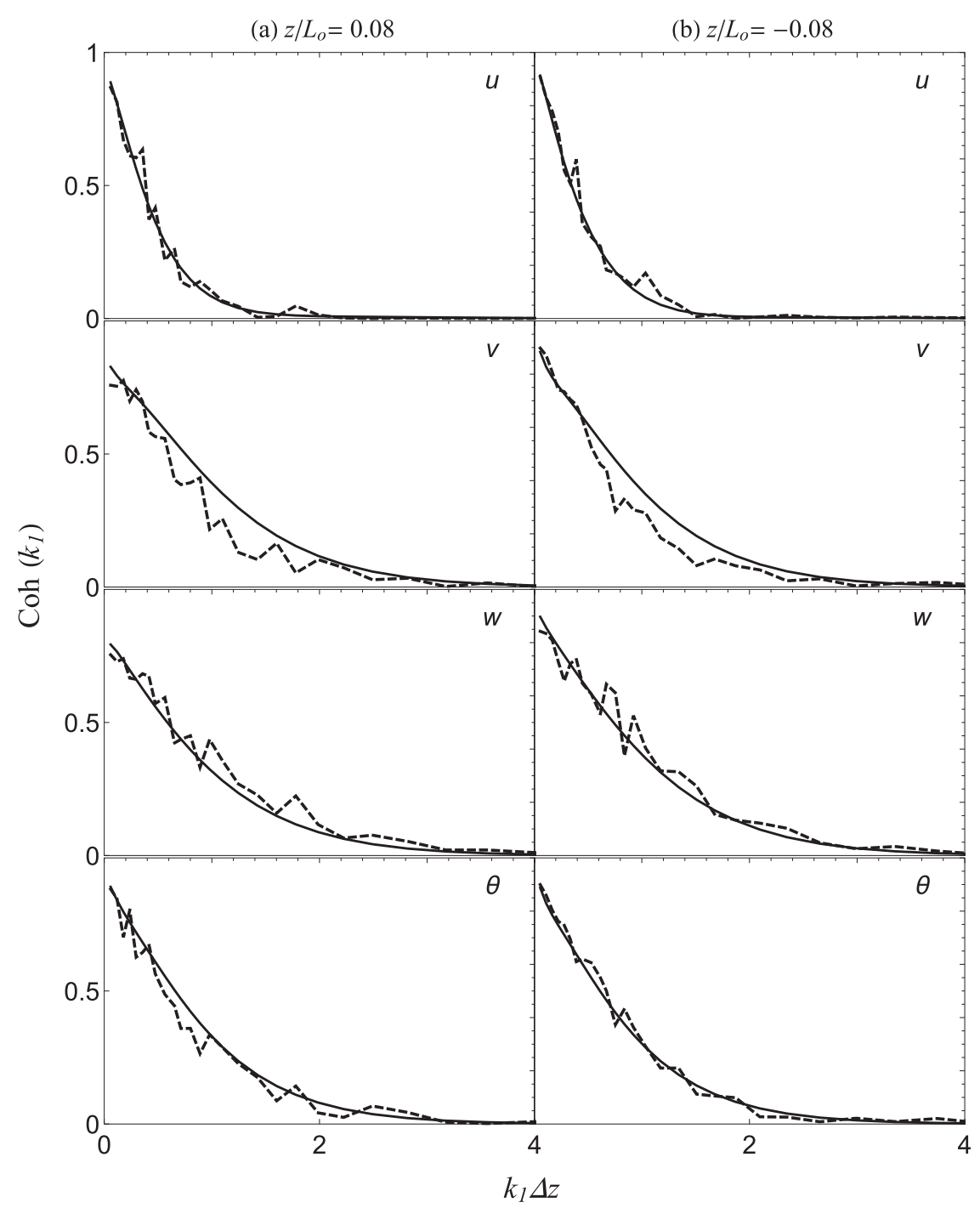

FIG. 10. Coherence predictions for (a) stable $\left(z / L_{o}=0.08\right)$ and (b) unstable $\left(z / L_{o}=-0.08\right)$ stratification are shown with the observations (dashed ragged lines) for vertical separation $\Delta z=1 \mathrm{~m}$.

The model coherences are independent of $\alpha \varepsilon^{2 / 3}$, which follows from (14), (15), and (45).

From Figs. 15 and 16 of the cross-spectral phases, it can be noted that the phase of temperature for vertical separation is larger than the phases in the streamwise and vertical velocity components. The model predicts the temperature phase to be on the order of the lateral velocity phase. Both the model and observed phases for horizontal separations are zero.

\section{c. Model stability parameters and Monin-Obukhov similarity theory}

Surface-layer similarity theory, originally introduced by Obukhov in 1946 (Obukhov 1946, 1971; Foken 2006; Businger and Yaglom 1971) and commonly called Monin-
Obukhov similarity theory (Wyngaard and Coté 1972) is based on dimensional analysis (Wyngaard 2010). A complete introduction to the foundation and application of the theory is beyond the scope of this paper, though we provide some relationships that can be arrived at via MOST, which has been shown to describe the surface-layer turbulence well in simple (flat, uniform surface) conditions. It parameterizes the effects of stability on dimensionless statistics in terms of $z / L_{o}$, with empirical forms determined for vertical profiles of, for example, mean wind speed, buoyant production, and viscous dissipation of TKE. Here we relate the model stability parameters in terms of MOST toward automated application of our model.

In steady-state (stationary) conditions, $\varepsilon$ and $\varepsilon_{\theta}$ are given by 
(a) $z / L_{o}=0.08$

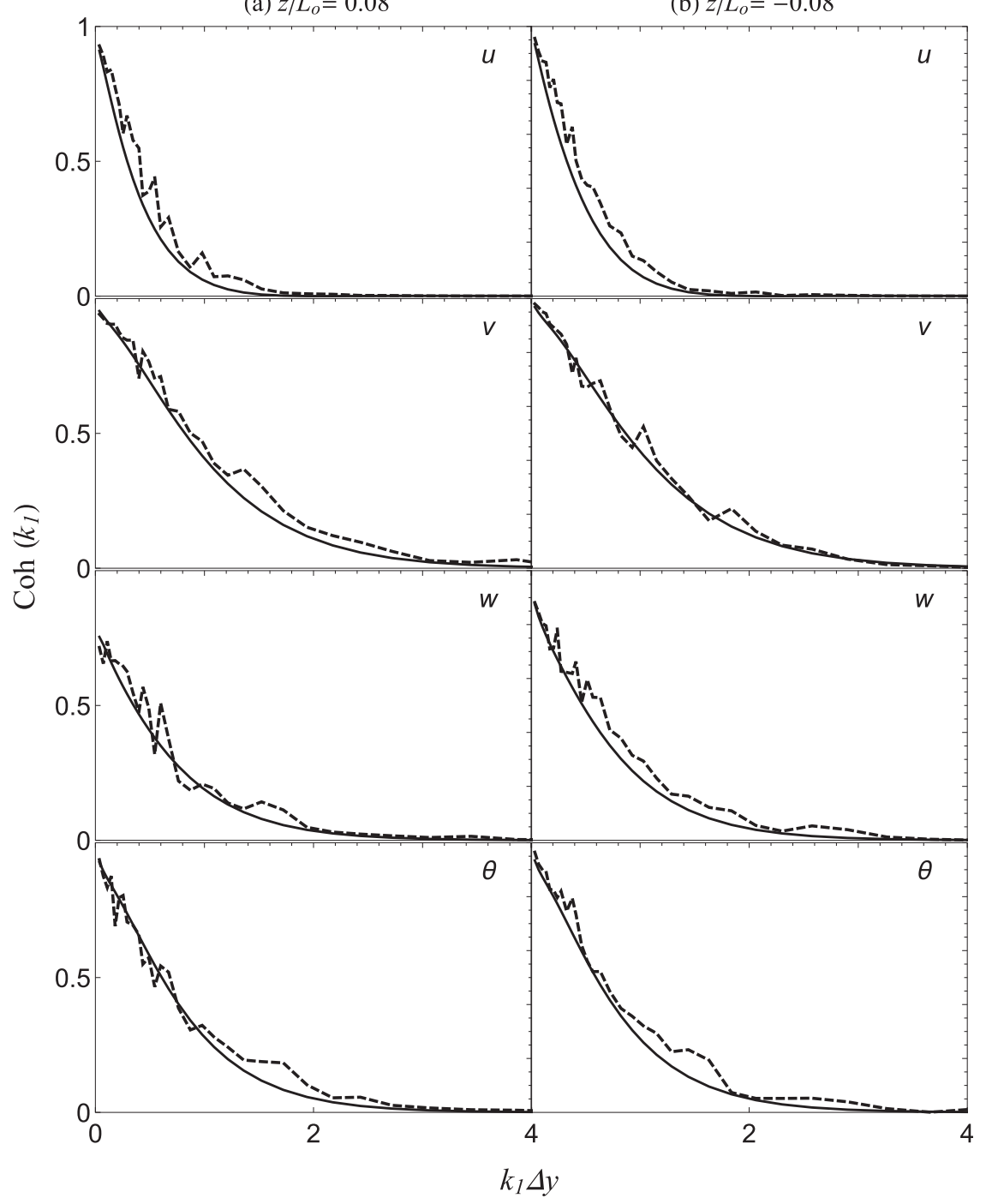

FIG. 11. Coherence predictions for (a) stable $\left(z / L_{o}=0.08\right)$ and (b) unstable $\left(z / L_{o}=-0.08\right)$ stratification are shown with the observations (ragged lines) for horizontal separation $\Delta y=0.63 \mathrm{~m}$.

$$
\varepsilon=-\left\langle u^{\prime} w^{\prime}\right\rangle\left(\frac{d U}{d z}\right)+\frac{g}{\bar{\theta}}\left\langle w^{\prime} \theta^{\prime}\right\rangle
$$

and

$$
\varepsilon_{\theta}=-\left\langle w^{\prime} \theta^{\prime}\right\rangle\left(\frac{d \bar{\theta}}{d z}\right)
$$

respectively. In homogeneous steady-state conditions, the dissipation rate of TKE is equal to the shear production [first term in (54)] plus buoyant production (second term). The shear production term is always positive, whereas the buoyant production term can be positive (source) or negative (sink), depending upon the vertical temperature flux $\left\langle w^{\prime} \theta^{\prime}\right\rangle$.
The sign of $\left\langle w^{\prime} \theta^{\prime}\right\rangle$ is generally opposite of the sign of the temperature lapse rate. By using the above relationships in (43), we get

$$
\eta_{\theta}=\frac{\mathrm{Ri}}{\mathrm{Ri}_{f}^{-1}-1},
$$

where $\mathrm{Ri}_{f}$ is the flux Richardson number and can be given as (Kaimal and Finnigan 1994)

$$
\begin{aligned}
& \mathrm{Ri}_{f}=\frac{(g / \bar{\theta})\left\langle w^{\prime} \theta^{\prime}\right\rangle}{\left\langle u^{\prime} w^{\prime}\right\rangle(d U / d z)} \\
& \simeq \frac{K_{h}}{K_{m}} \mathrm{Ri}
\end{aligned}
$$


(a) $z / L_{o}=0.014$

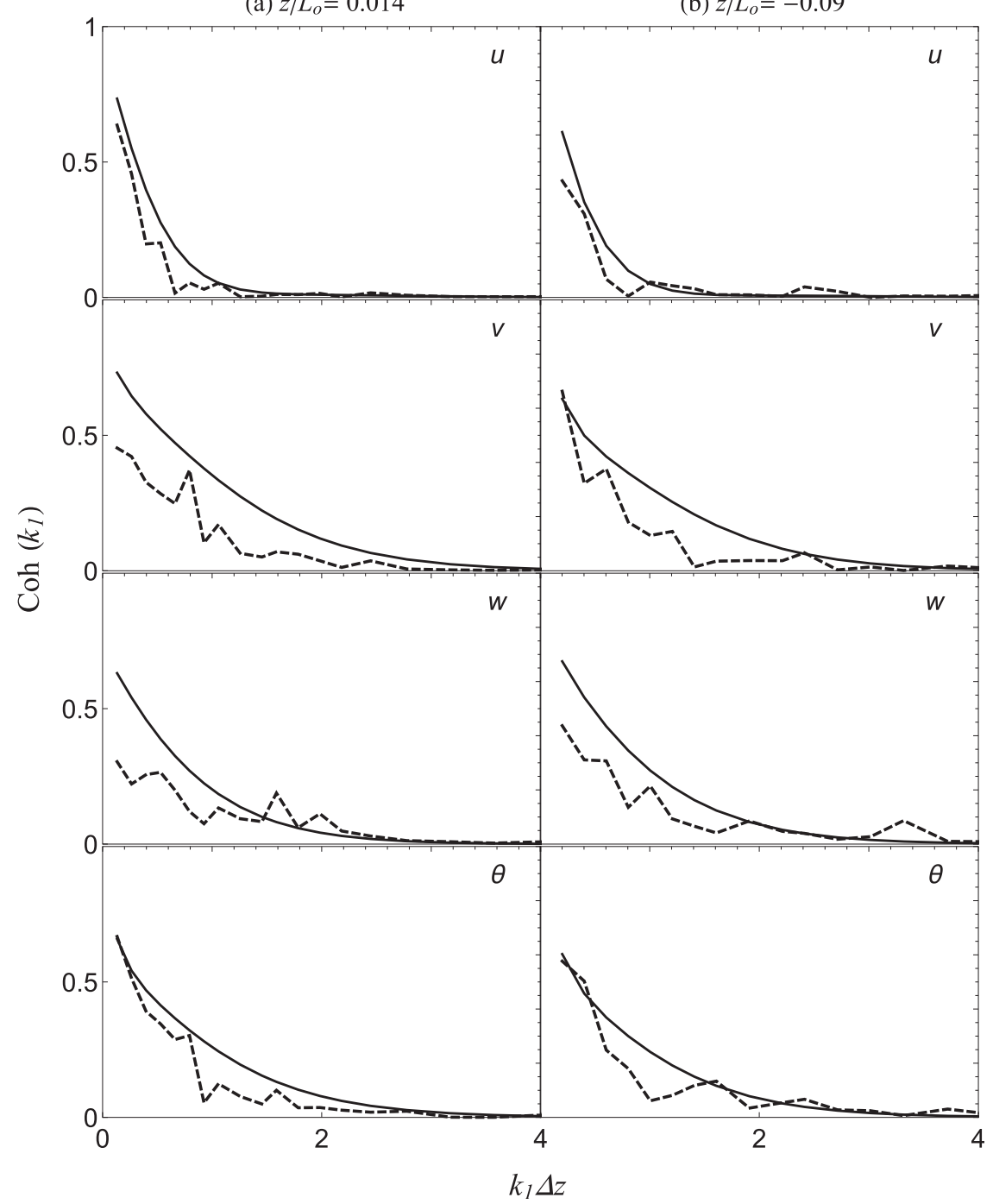

FIG. 12. Model coherence compared with observations for (a) $z / L_{o}=0.014$ and (b) $z / L_{o}=-0.09$ at $\Delta z=4 \mathrm{~m}$. Model coherences are shown by smooth lines, and the observed coherences are ragged lines.

where $K_{m}$ and $K_{h}$ are the turbulent exchange coefficients for momentum and heat, respectively. One can also find analogous empirically determined MOST relationships for Ri and $K_{m} / K_{h}$ as in Kaimal and Finnigan (1994):

$\mathrm{Ri}=\left\{\begin{array}{lr}z / L_{o}, & -2 \leq z / L_{o} \leq 0 \\ \left(z / L_{o}\right)\left(1+5 z / L_{o}\right)^{-1}, & 0 \leq z / L_{o} \leq 1\end{array}\right.$

and

$\frac{K_{m}}{K_{h}}=\left\{\begin{array}{lr}\left(1+16\left|z / L_{o}\right|\right)^{-1 / 4}, & -2 \leq z / L_{o} \leq 0 \\ 1, & 0 \leq z / L_{o} \leq 1 .\end{array}\right.$
From the above relationships, the $\eta_{\theta}$ parameter can be expressed from (56) as

$$
\eta_{\theta}\left(z / L_{o}\right)=\frac{\mathrm{Ri}^{2}}{\left(K_{m} / K_{h}\right)-\mathrm{Ri}} ;
$$

determinations of such MOST forms and comparison to field data can be found in, for example, Businger et al. (1971).

We compare the stability parameter Ri obtained from the spectral fits of the model to the data, with the gradient Richardson number as a function of $z / L_{o}$ in (59) determined by MOST, and shown in Fig. 19a. Here we have fitted the model spectra and cospectra to the more 


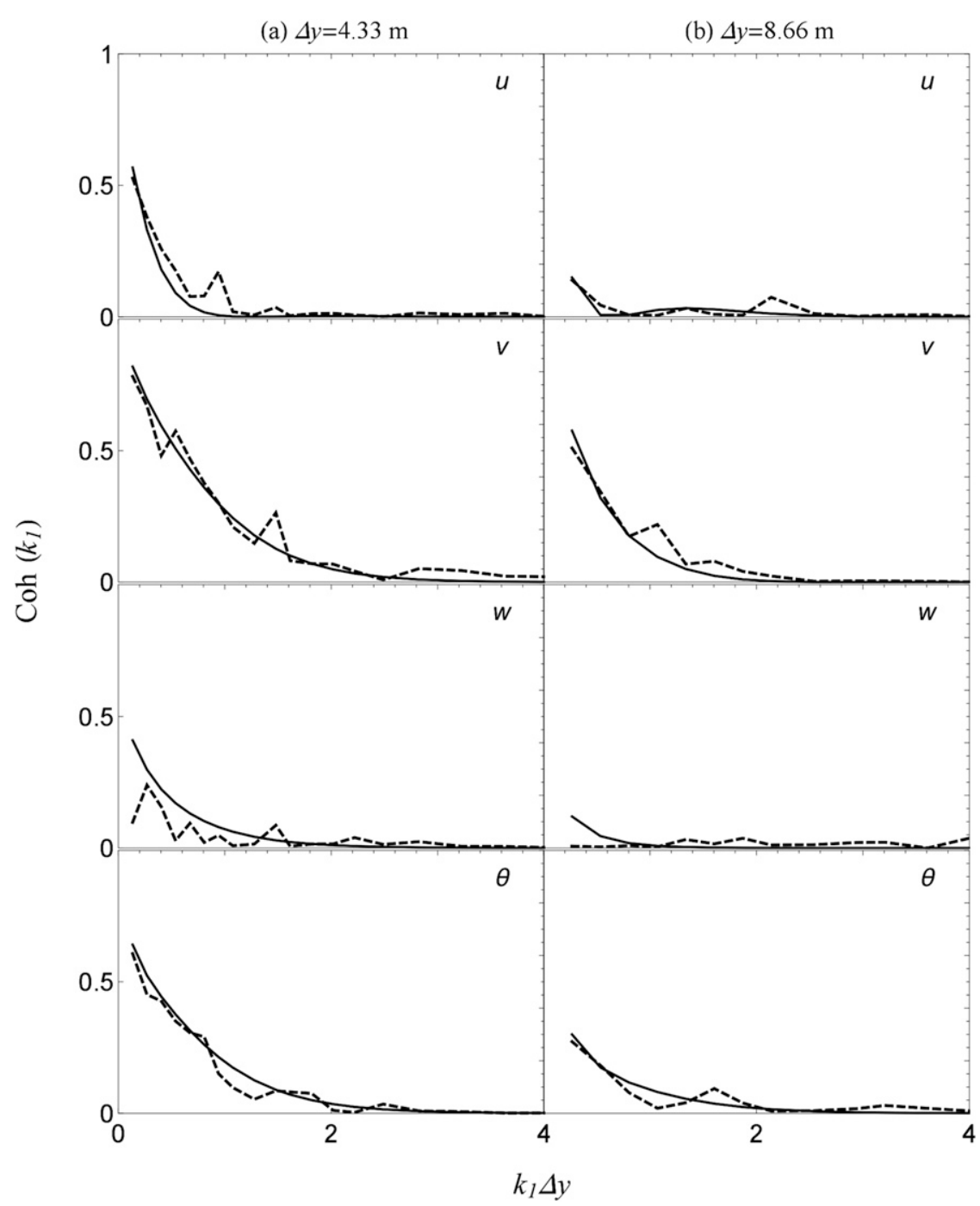

FIG. 13. Model coherences (smooth lines) compared with observations (ragged lines) for $z / L_{o}=0.014$ at (a) $\Delta y=4.33$ and (b) $\Delta y=8.66 \mathrm{~m}$.

stable cases, for $z / L_{o}$ values up to 1.74 . The gradient Richardson numbers from the model (spectral) fits are roughly 5/8 times the Ri values from MOST, and $\sim 5 / 7$ times Ri calculated from the measurements as shown in Fig. 19b. We calculate Ri from the data using (32), where we take $g=9.81 \mathrm{~m} \mathrm{~s}^{-2}$ and $\bar{\theta}=288 \mathrm{~K}$, and the shear and temperature lapse rate are calculated by taking the finite difference in the averaged mean wind speed $U$ and averaged mean potential temperature between $s$ and $d$ arrays (divided by the vertical distance $\Delta z=\left|z_{s}-z_{d}\right|$ ). The gradient Richardson numbers obtained from the model fits are compared with the flux Richardson number $\left(\mathrm{Ri}_{f}\right)$ obtained from MOST [using (58)-(60)] and with $\mathrm{Ri}_{f}$ calculated from the observations [(57)] in Figs.20a and 20b, respectively. In the limit where similarity theory applies,
$K_{m} / K_{h}=1$ and $\mathrm{Ri}_{f} \approx \mathrm{Ri}$ in the stable $\mathrm{ABL}$, whereas the ratio between the flux Richardson number from MOST and the gradient Richardson number from the model fits is $\sim 1.5$ [Fig. 20a] and that between observed $\mathrm{Ri}_{f}$ and $\mathrm{Ri}$ from RDT is $\sim 1.6$ [Fig. 20b]. In the stable regime, data follow similarity theory reasonably in the limit where $\mathrm{Ri}_{f} \approx \mathrm{Ri}$, as shown in Fig. 20c.

The $\eta_{\theta}$ parameters from the model fits are compared with $\eta_{\theta}$ estimated as a similarity function from (61), and also calculated from the measurements using (43), (54), and (55); these are shown in Figs. 21a and 21b, respectively. Here, the model parameter $\eta_{\theta}$ obtained from spectral fits is smaller by a factor of $\sim 1 / 3$, compared to that given by (61) and that obtained from the data. Basically, the model does not appear to be 


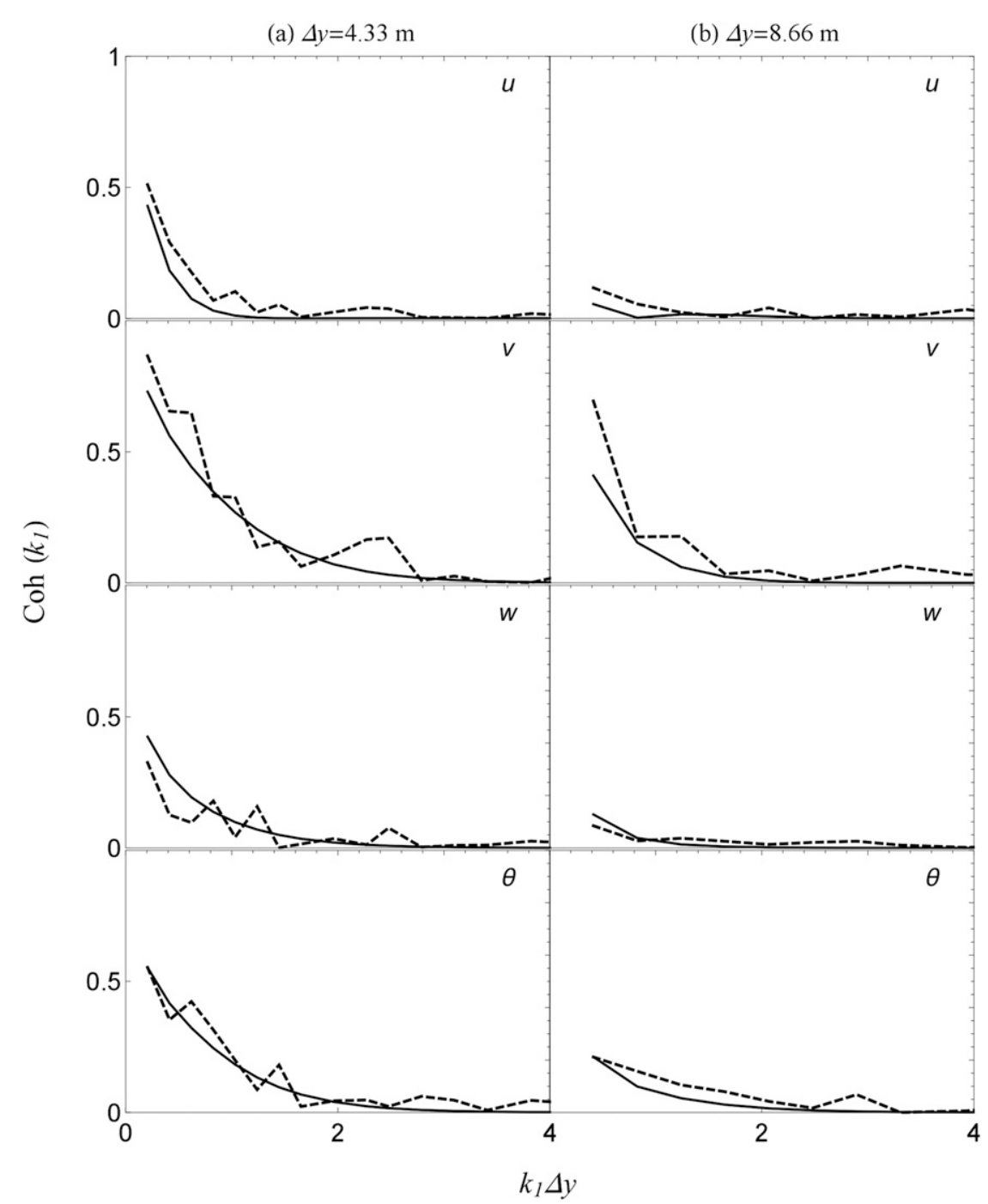

FIG. 14. Model coherences (smooth lines) compared with observations (ragged lines) at (a) $\Delta y=4.33$ and (b) $\Delta y=8.66 \mathrm{~m}$ for $z / L_{o}=-0.09$.

completely consistent with the empirical functions of MOST. The comparisons between MOST and the data for $\mathrm{Ri}, \mathrm{Ri}_{f}$, and $\eta_{\theta}$ are shown in Figs. 22a-c, respectively. From MOST, $\left\{\mathrm{Ri}, \mathrm{Ri}_{f}, \eta_{\theta}\right\}$ are calculated as functions of $z / L_{o}$ [using (59) for $\mathrm{Ri},(58)-(60)$ for $\mathrm{Ri}_{f}$, and (61) for $\left.\eta_{\theta}\right]$; whereas those from the data are calculated using (32) for $\mathrm{Ri},(57)$ for $\mathrm{Ri}_{f}$, and (43), (54), and (55) for $\eta_{\theta}$. It can be shown from Fig. 22 that the HATS data agree reasonably well with similarity theory.

The main discrepancy between the RDT model and MOST is that the RDT model is based on the assumption where the nonlinear fluctuation-fluctuation terms are neglected [second term in (22)], whereas MOST is based on the dimensional analysis of surfacelayer gradients (velocity, temperature, etc.), with their physical behavior provided by empirical relationships (Kaimal and Finnigan 1994). Another difference is that the RDT model assumes homogeneity in all three directions, whereas the MOST assumes homogeneity on the two horizontal directions only. We know that the assumption of homogeneity in the vertical direction breaks down (particularly at lower frequencies, as discussed earlier) where the neglected fluctuation-fluctuation term in (22) might become important.

\section{d. Turbulence anisotropy}

From the Reynolds stress tensor $R_{i j}$ it is possible to investigate the statistical deviation of the turbulence from isotropy and its three-dimensional character, via the anisotropy tensor defined by 


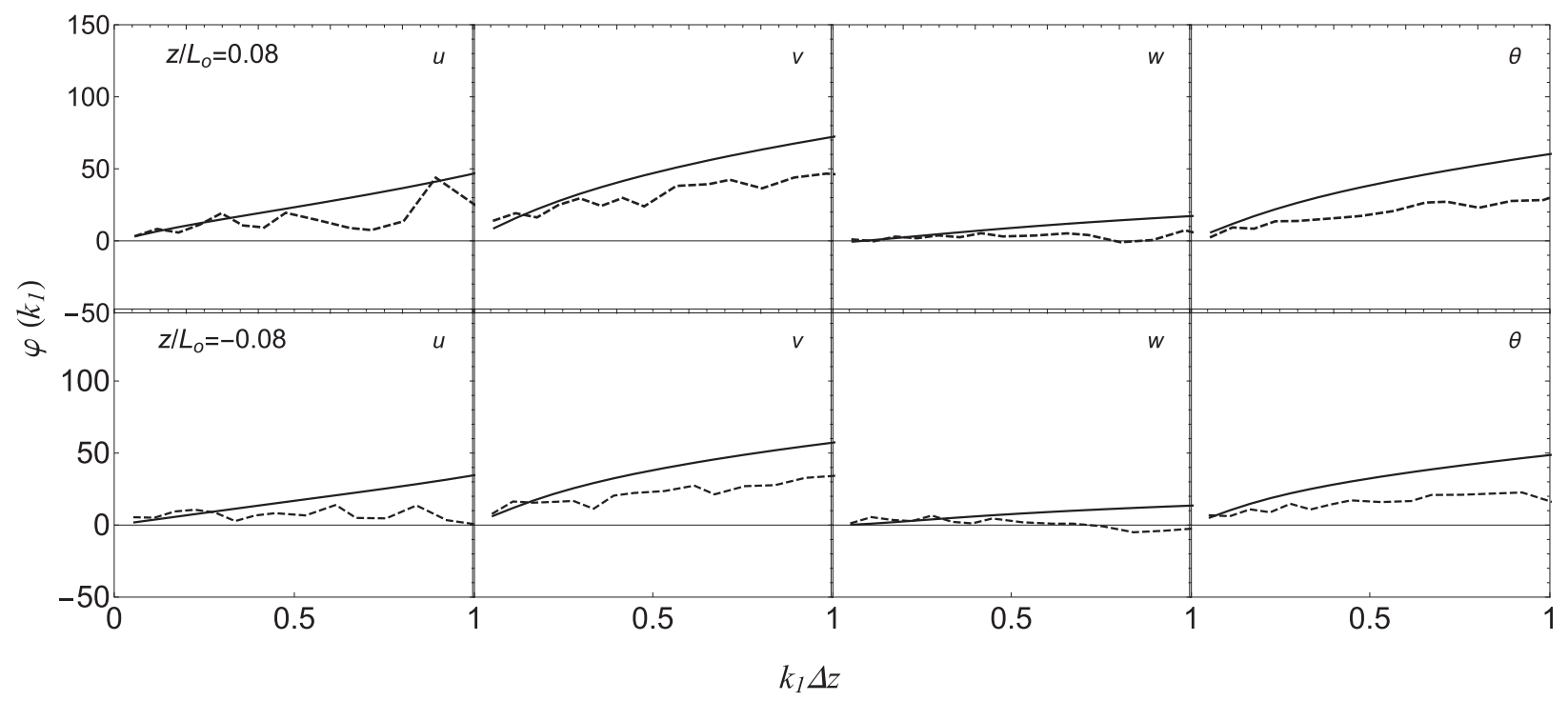

FIG. 15. Phases $\left({ }^{\circ}\right)$ in the modeled cross-spectra (smooth lines) for (top) stable $\left(z / L_{o}=0.08\right)$ and (bottom) unstable $\left(z / L_{o}=-0.08\right)$ stratification are shown with the observed (ragged lines) for vertical separation $\Delta z=1 \mathrm{~m}$.

$$
b_{i j}=\frac{R_{i j}(0)}{R_{k k}(0)}-\frac{1}{3} \delta_{i j}
$$

again, $R_{k k}(0)$ is simply twice TKE, following (10). As given in Pope (2000), two variables $\gamma$ and $\zeta$ can be defined from $b_{i j}$ and invariants of the turbulent field:

$$
\begin{aligned}
\gamma^{3} & =\frac{1}{2} \lambda_{1} \lambda_{2} \lambda_{3} \quad \text { and } \\
\zeta^{2} & =-\frac{1}{3}\left(\lambda_{1} \lambda_{2}+\lambda_{2} \lambda_{3}+\lambda_{3} \lambda_{1}\right) .
\end{aligned}
$$

The matrix $\mathbf{b}$ can be transformed into principal axes via the eigenvalues $\lambda_{1}, \lambda_{2}$, and $\lambda_{3}$, where off-diagonal terms become zero: that is, $b_{i i}=\lambda_{1}+\lambda_{2}+\lambda_{3}=0$, so $\lambda_{3}=-\left(\lambda_{1}+\lambda_{2}\right)$.

The state of the anisotropy tensor can be represented in a $\gamma-\zeta$ plane, called the Lumley triangle (Pope 2000), where limiting states of turbulence (and the Reynolds stress tensor) correspond to edges of the "triangle" in the plane, as shown in Fig. 23. The origin $(\gamma=\zeta=0$, bottom of the Lumley triangle) represents isotropic turbulence, where $b_{i j}=0$. Here we present analysis for several stable

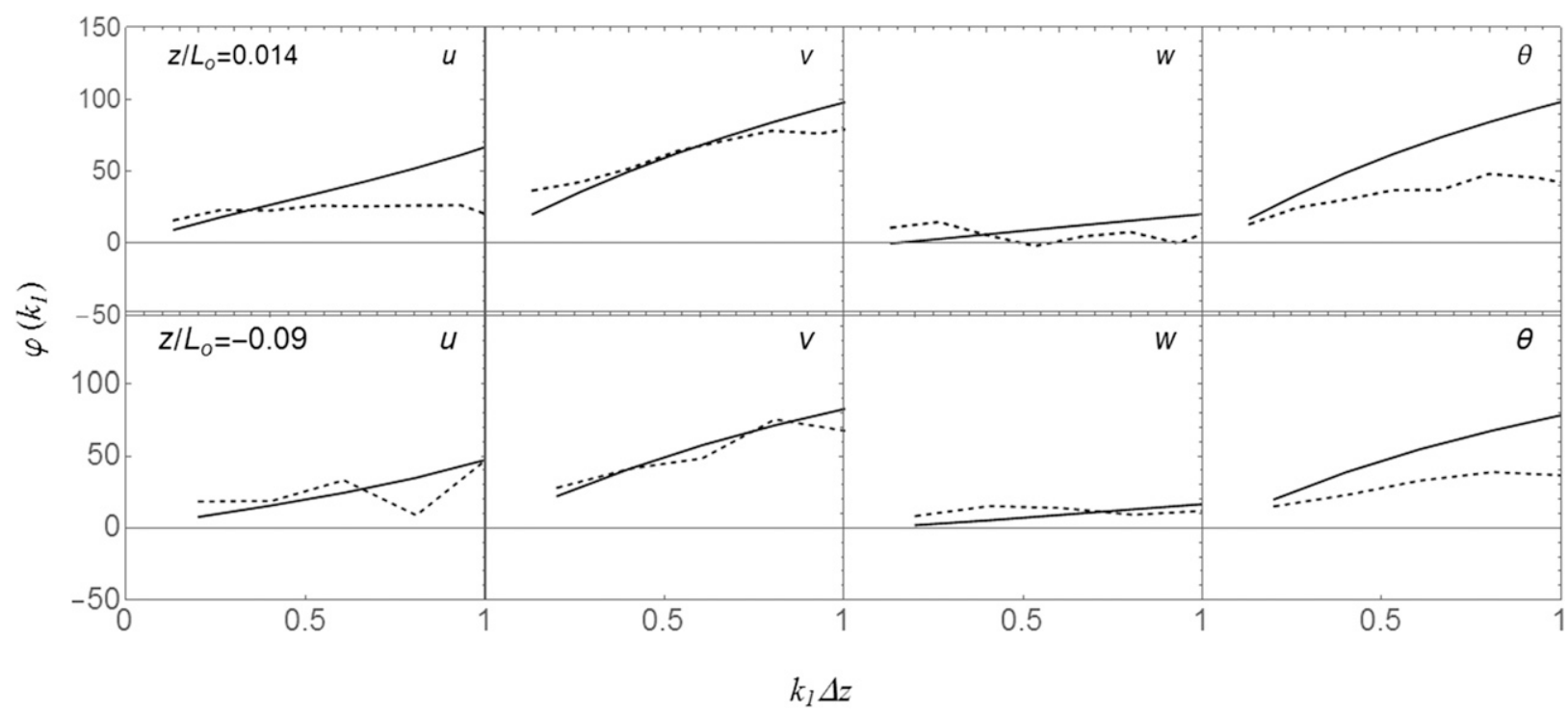

FIG. 16. Modeled phases (smooth lines) compared with observations (dashed lines) for $z / L_{o}=0.014$ and $z / L_{o}=-0.09$ at $\Delta z=4 \mathrm{~m}$. 
$\mathrm{s}$

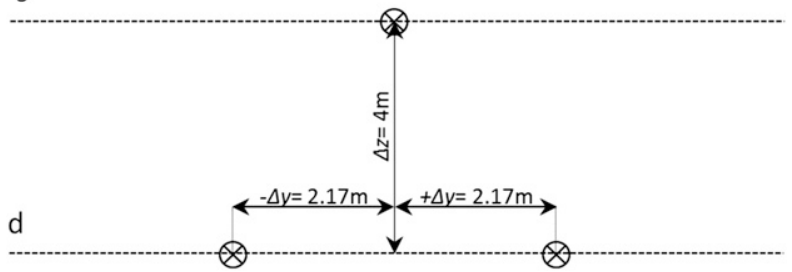

FIG. 17. Sketch for the coherence calculations in Fig. 18 to test the left-right symmetry. Coherences are calculated with respect to the middle sonic in the $s$ array.

cases (I) and a single unstable stratification case (II) with $z / L_{o}=-0.03$ at $z=6 \mathrm{~m}$. All the stable cases fall on the right side in the Lumley triangle, except for the observations with $z / L_{o}=0.13$ (solid diamond), which fall close to the left border of the Lumley triangle. The latter axisymmetric kind of turbulence is typical of convectiondominated regimes, where one eigenvalue of $b_{i j}$ is determined from vertical fluctuations (and smaller than the other two, equal eigenvalues); indeed, the observed $b_{i j}$ and corresponding $(\gamma, \zeta)$ for the unstable $z / L_{o}=-0.03$ case falls on this side of the Lumley triangle. However, the corresponding point from the model for the strongest stable case $\left(z / L_{o}=0.13\right.$, open diamond) falls on the opposite side, as do all of the model predictions, including the unstable case. These predictions, and the neutral and remaining stable-case observations, fall near the right leg of the Lumley triangle; they exhibit a rodlike turbulence shape that is more typical of neutral and weakly stable conditions driven by shear-generated turbulence (Pope 2000). The model is able to represent the latter axisymmetric turbulence having one large eigenvalue but does not reproduce the other axisymmetric regime (i.e., the left border of the Lumley triangle). We postulate that the model cannot replicate the convective-type axisymmetric turbulence structures because it lacks the (direct) effect of the surface; ongoing work includes incorporating the surface-blocking extension to the model of M94 in the buoyancy-affected spectral tensor to allow a more realistic RDT reproduction of such turbulent regimes.

\section{Conclusions}

A spectral velocity tensor model including buoyancy, via a uniform vertical temperature gradient, is developed. This development is based on the RDT applications in M94 and HH04. The model assumes zero initial temperature fluxes. The initial fluctuations in velocity components are introduced through the von Kármán energy spectrum for isotropic turbulence, and the fluctuations in the temperature are initiated by taking a potential energy spectrum proposed by Kaimal and Finnigan (1994). The model assumes mean uniform shear and lapse rate, where the eddies get distorted spatially and temporally by the mean shear, as can be seen from the three-dimensional wavenumber vector. A mechanism is developed through the RDT equations with the initial conditions, which creates the correlations between the velocity components and maintains correlations among velocity components and the temperature via mean uniform shear and lapse rate. This mechanism is time limited by replacing the time variable with the wavenumber-dependent eddy lifetime of M94, giving a statistical description of the stationary process whereby turbulence is generated and turbulent eddies are stretched over a finite extent. This "picture" can be described via five parameters that the model contains: $\alpha \varepsilon^{2 / 3}$, $L, \Gamma, \mathrm{Ri}$, and $\eta_{\theta}$. These parameters are obtained by fitting the one-dimensional spectra and cospectra with the data.

The model results are compared with the atmospheric surface-layer data obtained from HATS. The results show that the model is able to regenerate velocity and temperature spectra within reasonable agreement and predict the cospectrum between the longitudinal and the vertical velocity and that between the temperature and the vertical velocity, thereby predicting the vertical temperature fluxes for the given stratifications in terms of $z / L_{o}$. The model systematically underestimates the cospectra between the temperature and the horizontal velocity. On the stable side, the parameters $L$ and $\Gamma$ both decrease for increasing $z / L_{o}$, while the new stability parameters $\mathrm{Ri}$ and $\eta_{\theta}$ unsurprisingly increase with $\left|z / L_{o}\right|$. The streamwise temperature flux is observed to be larger than the vertical temperature flux, while the ratio between the two decreases with stronger stable stratification. The turbulence as prescribed by the model seemed more anisotropic in the stable than the unstable situations; additionally, the RDT-induced shear is larger in stable than in unstable conditions, while the turbulence becomes more and more isotropic in nature as stability approaches a neutral state. The buoyancy effect introduced in the model is also projected via the length scale $L$ parameter in the model. Length scales corresponding to the spectral peaks of the $\theta$ spectrum and $w \theta$ cospectrum coincide with each other, and length scales for $u \theta$ are larger than those for $\theta$ and $w \theta$ observed in both stable and unstable ABLs.

For stable stratification, the model predicts a slope closer to -3 for the $u \theta$ cospectrum, also seen in the data. This supports the theoretical prediction by Wyngaard and Coté (1972), while an averaged slope of $-5 / 2$ for stable stratification was noted in Kaimal et al. (1972). The data shows a slope of $-7 / 3$ for unstable cases, which was also noted in Wyngaard and Coté (1972) in their data at a higher height. 
(a) $z / L_{o}=0.014$

(b) $z / L_{o}=-0.09$

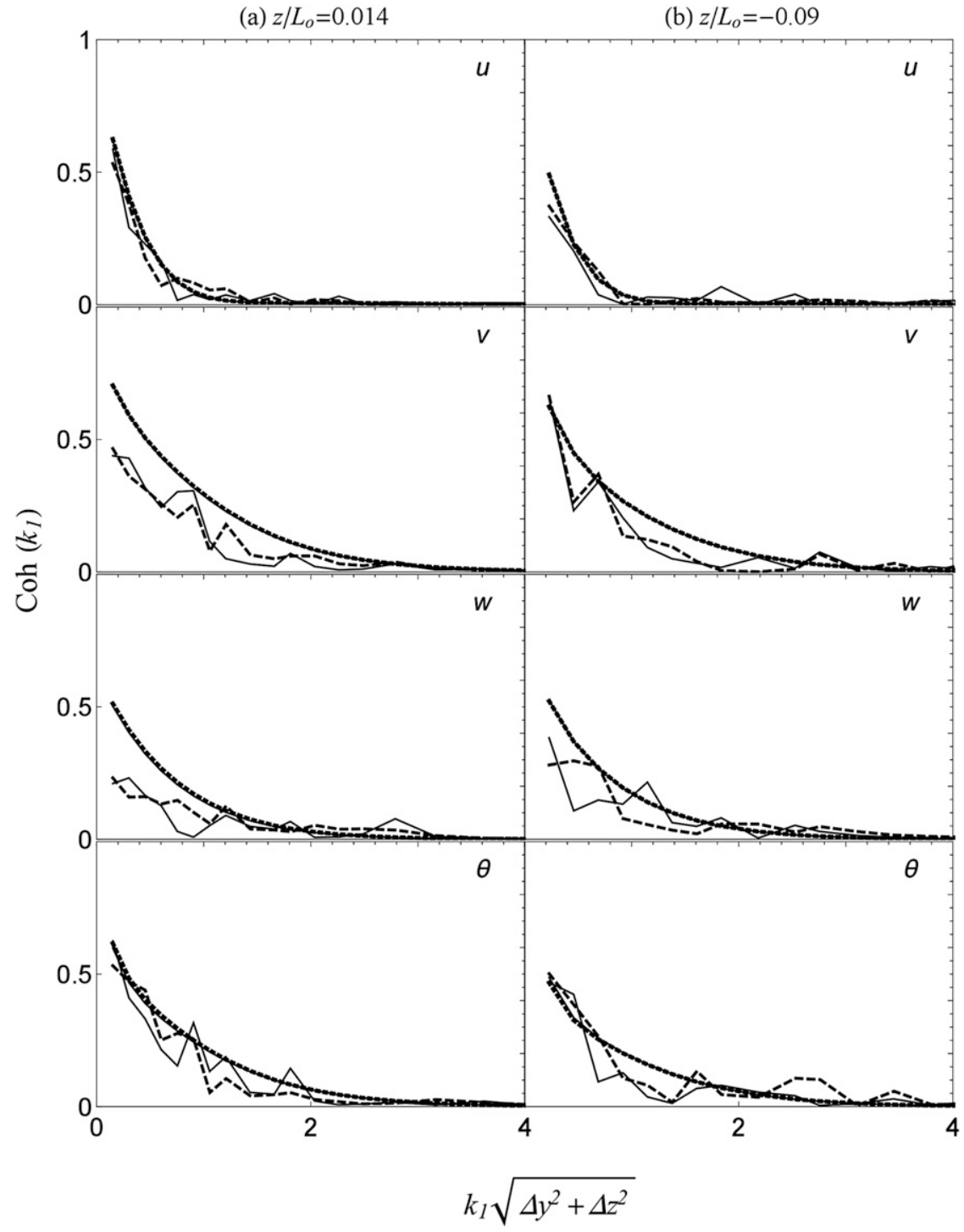

FIG. 18. Model coherence (smooth lines) compared with observations (ragged lines) for (a) $z / L_{o}=0.014$ and (b) $z / L_{o}=-0.09$, with separations $\pm \Delta y=2.17 \mathrm{~m}$ and $\Delta z=4 \mathrm{~m}$. Coherences for $+\Delta y$ are indicated by solid lines, whereas the dashed lines shows coherences for $-\Delta y$ separation.

The results from the modeled cross-spectra are compared with the data in terms of coherence and phases for vertical and lateral separations. The results show that the model performs relatively better for lateral than for vertical separations; the model overestimates coherence more for vertical separations, perhaps because of the flow's inhomogeneity in the vertical direction. The measurements support the left-right symmetry of the model, tested via coherence. The phases in the modeled cross-spectra are compared with the phases from the measured time series, which show that the phase of temperature for vertical separation is larger than the phases in the streamwise and vertical velocity components, and the phases for horizontal separation are zero.

The ratio between the gradient Richardson number given by MOST and that from fitting the spectral tensor output is approximately equal to 1.6 , while the analogous ratio of the $\eta_{\theta}$ parameter gives a value close to 0.3 . This shows that, as per the HATS data, the model is not completely in agreement with (some of) the empirical MOST functions. Finally, we conclude that the model is able to represent the anisotropic nature of the turbulence in the limit of shear-driven axisymmetry (with one eigenvalue of the anisotropic stress tensor larger than the 
(a)

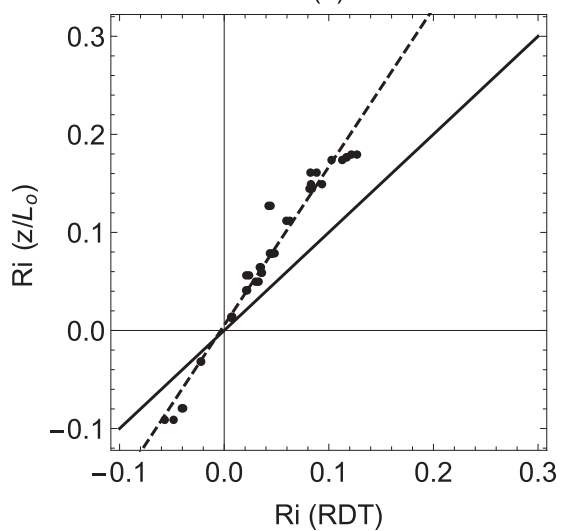

(b)

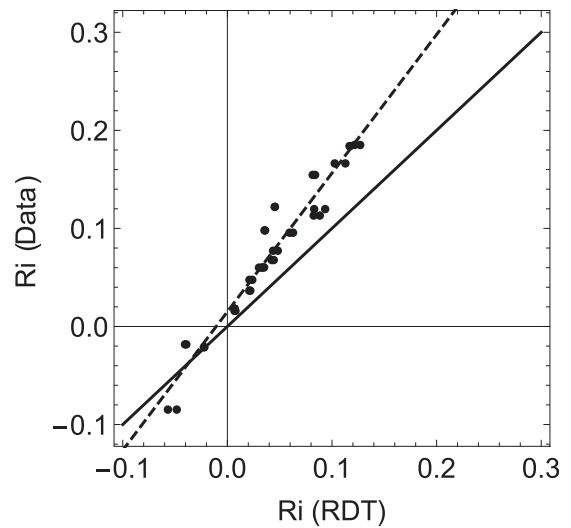

FIG. 19. Gradient Richardson number obtained from the model fits compared with (a) Ri as function of $z / L_{o}$ via (59) and (b) Ri from the data. Dashed lines show linear fits having slope of $\sim 1.6$ in (a) and $\sim 1.4$ in (b), with a 99\% confidence level. Solid lines are slopes 1:1 in both graphs.

other two, which are equal). However, the model cannot mimic the turbulence shapes for the axisymmetric regime where one eigenvalue is smaller, as in free-convection conditions. As described in the previous section, we think that this discrepancy for unstable conditions is likely due to the lack of surface-induced blocking in the model. Finally, the model can also be improved with the extension of the work of de Maré and Mann (2016) in mind.

Acknowledgments. This work has been funded by the Norwegian Centre for Offshore Wind Energy (NORCOWE) under Grant 193821/S60 from the Research Council of Norway (RCN). The Danish EUDP project "Impact of atmospheric stability conditions on wind farm loading and production" under Contract 64010-0462 is acknowledged for financial support of this study. We are also obliged to the COMWIND project funded by the Danish Council of Strategic Research (DSF Contract 09-067216). The authors thank Senior Researcher Dalibor Cavar (DTU Wind Energy) for assisting with calculations on DTU's cluster. The authors are also thankful to the anonymous reviewers whose critical comments and suggestions have improved the manuscript in many ways. Finally, we are grateful to Dr. Tom Horst at the National Center for Atmospheric Research for making the HATS data available to us.

\section{APPENDIX}

\section{From Isotropic to Anisotropic Tensor}

We combine the isotropic-spectral velocity tensor and the three-dimensional temperature spectrum provided (a)

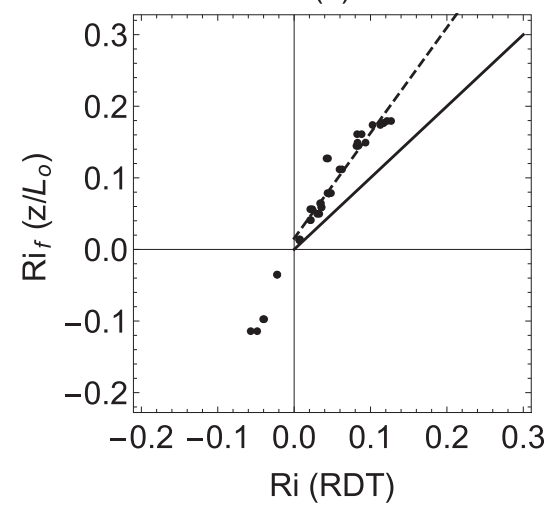

(b)

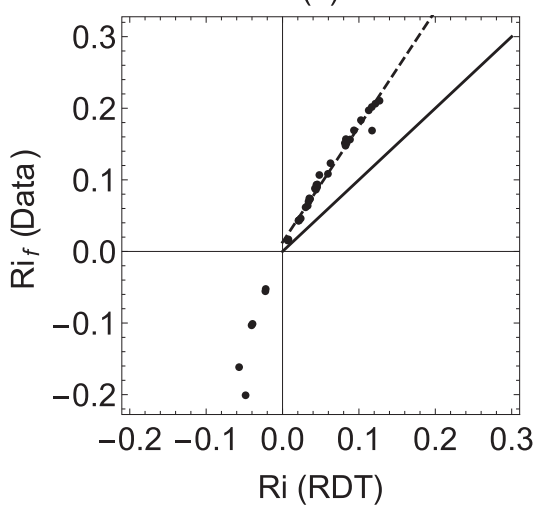

(c)

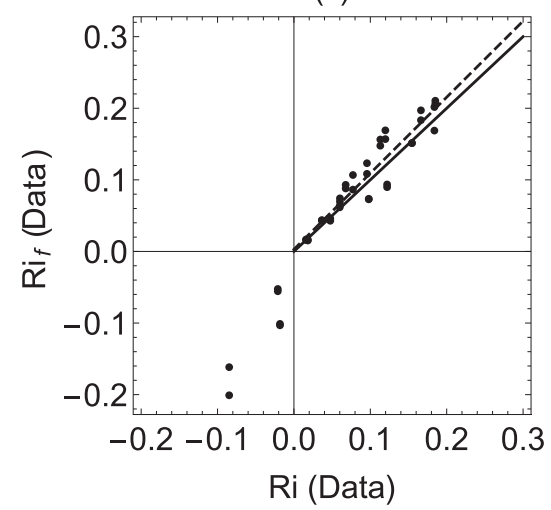

FIG. 20. Ri obtained from the model fits compared with $\mathrm{Ri}_{f}(\mathrm{a})$ as a function of $z / L_{o}$ and (b) obtained from the data. (c) $\mathrm{Ri}_{f}$ obtained from the data, is compared with Ri calculated from the data. Dashed lines are linear fits in a stable regime having slope of $\sim 1.5$ in (a), $\sim 1.6$ in (b), and $\sim 1.06$ in (c). Solid lines show the 1:1 slope in all graphs. 
(a)

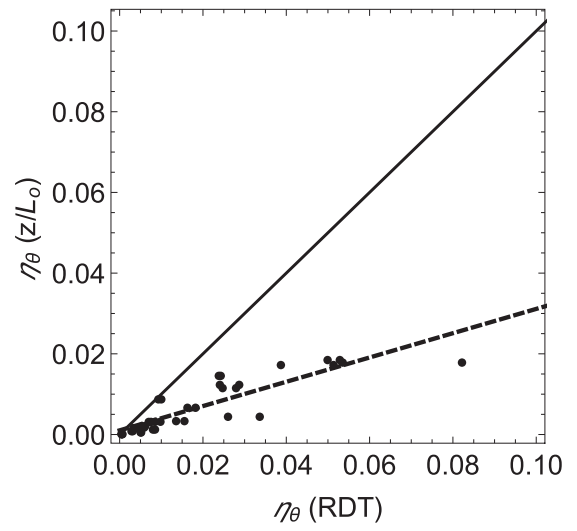

(b)

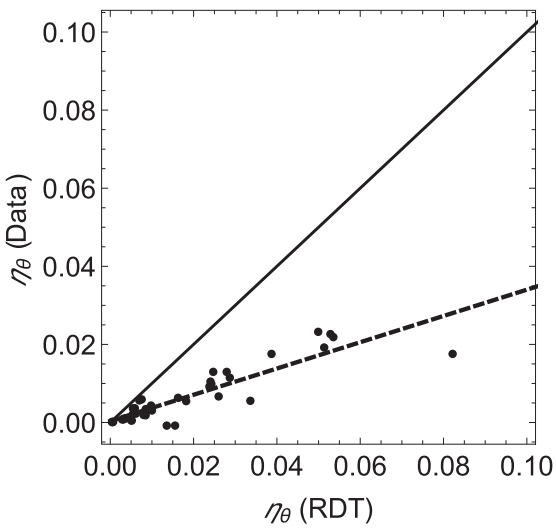

FIG. 21. The model parameter $\eta_{\theta}$ compared with that calculated (a) as a function of $z / L_{o}$ via (59)-(61) and (b) from measurements. Dashed lines are linear fits obtained from the data shown in the graphs with a $99 \%$ confidence level and have slopes of $\sim 0.3$ in (a) and $\sim 0.33$ in (b). Solid lines are the 1:1 slopes.

in (34) and (41) and, by assuming the zero initial temperature fluxes, we obtain

$$
\boldsymbol{\Phi}\left(\mathbf{k}_{0}, 0\right)=d Z_{0} \cdot d Z_{0}^{\mathrm{T}}
$$

where

$d Z_{0}=\left(\begin{array}{cccc}0 & k_{3} & -k_{2} & 0 \\ -k_{3} & 0 & k_{1} & 0 \\ k_{2} & -k_{1} & 0 & 0 \\ 0 & 0 & 0 & \sqrt{k^{2} \frac{S^{\prime}(k)}{E(k)}}\end{array}\right) \sqrt{\frac{E(k)}{4 \pi k^{4}}}$.
From the definitions of $E(k)$ and $S^{\prime}(k), \boldsymbol{\Phi}\left(\mathbf{k}_{0}, 0\right)$ becomes a function of $\alpha \varepsilon^{2 / 3}, L$, and $\beta \eta_{\theta}$ and can be expressed as

$$
\boldsymbol{\Phi}\left(\mathbf{k}_{0}, 0\right) \equiv \boldsymbol{\Phi}\left(\mathbf{k}_{0}, \alpha \varepsilon^{2 / 3}, L, \beta \eta_{\theta}\right) .
$$

Let $\mathbf{A} \equiv \mathbf{A}(\mathbf{k}, \xi$, Ri) be the solution matrix of the RDT equations in (30) obtained for the set of initial conditions $\mathbf{I}_{0}$, where

$$
\mathbf{I}_{0}=\left(\begin{array}{llll}
1 & 0 & 0 & 0 \\
0 & 1 & 0 & 0 \\
0 & 0 & 1 & 0 \\
0 & 0 & 0 & 1
\end{array}\right)
$$

Therefore, the spectral tensor $\boldsymbol{\Phi}(\mathbf{k}, \xi)$ formed from the solution of the RDT equations can be given as (a)

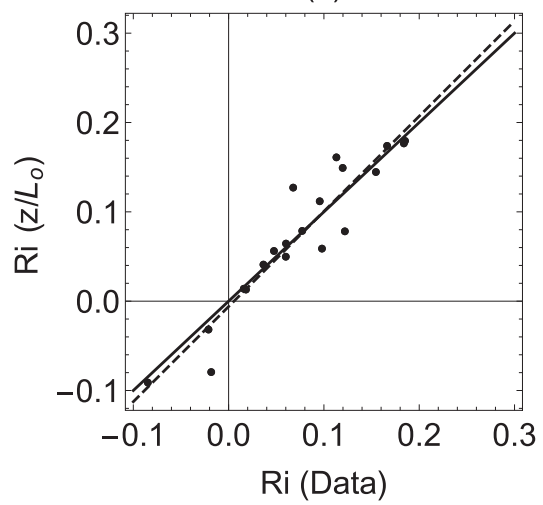

(b)

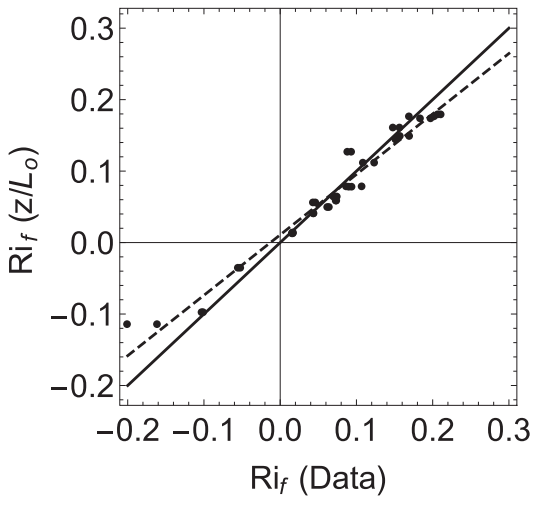

(c)

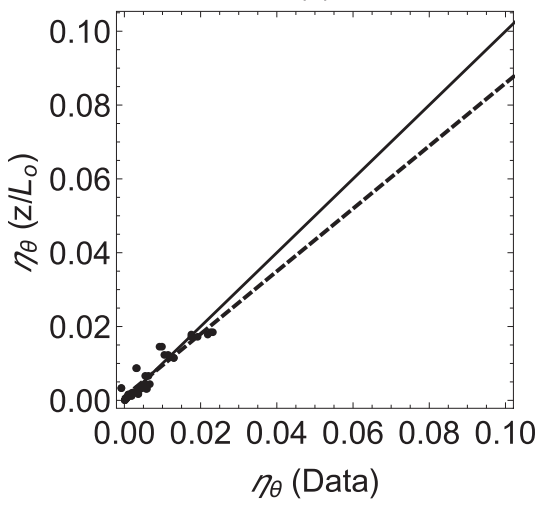

FIG. 22. (a) $\mathrm{Ri}$, (b) $\mathrm{Ri}_{f}$, and (c) $\eta_{\theta}$ calculated from the data are compared with those as a function of $z / L_{o}$ via MOST. Dashed lines are linear fits with slopes of $\sim 1.06$ in (a), $\sim 0.9$ in (b), and $\sim 0.85$ in (c). Solid lines are the 1:1 slopes. 


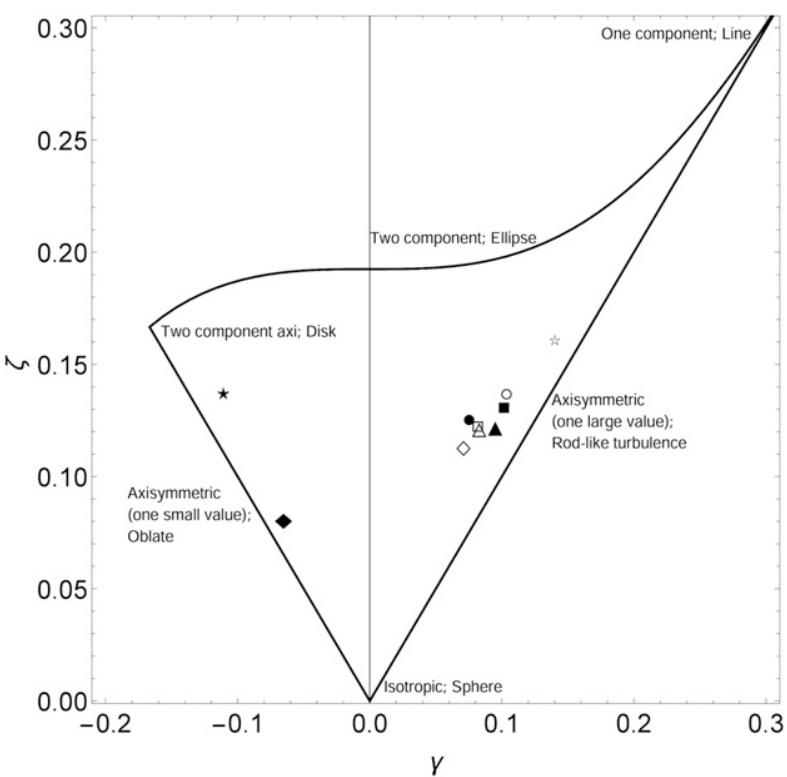

FIG. 23. Statistical turbulent shapes through invariants in the Lumley triangle (Pope 2000). Symbols correspond to $z / L_{o}$ as follows: circles are for $z / L_{o}=0.015$; triangles are for $z / L_{o}=0.05$; squares are for $z / L_{o}=0.095$; diamonds are for $z / L_{o}=0.13$; and stars are for $z / L_{o}=-0.03$. Filled symbols are derived from the data, and empty symbols are from the model.

$$
\boldsymbol{\Phi}(\mathbf{k}, \xi)=\mathbf{A} \cdot \frac{\boldsymbol{\Phi}\left(\mathbf{k}_{0}, 0\right)}{J} \cdot \mathbf{A}^{\mathrm{T}},
$$

where $J=\partial\left(k_{1}, k_{2}, k_{3}\right) / \partial\left(k_{1}, k_{2}, k_{30}\right)$ is the Jacobian. From the above equation and (A3),

$$
\boldsymbol{\Phi}(\mathbf{k}, \xi) \equiv \boldsymbol{\Phi}\left(\mathbf{k}, \alpha \varepsilon^{2 / 3}, L, \xi, \mathrm{Ri}, \beta \eta_{\theta}\right) .
$$

From (35), (42), (A1), and (A5), it can be proven that

$$
\begin{aligned}
& \boldsymbol{\Phi}\left(\mathbf{k}, \alpha \varepsilon^{2 / 3}, L, \xi, \mathrm{Ri}, \beta \eta_{\theta}\right) \\
& \quad=\alpha \varepsilon^{2 / 3} L^{11 / 3} \boldsymbol{\Phi}\left(\mathbf{k} L, 1,1, \xi, \mathrm{Ri}, \beta \eta_{\theta}\right) .
\end{aligned}
$$

For simplicity, we take $J=1$ as in, for example, Mann (1994a, 2000), assuming that it might not affect the spectra much; but at lower wavenumbers, detailed investigations need to be done.

\section{REFERENCES}

Batchelor, G. K., 1953: The Theory of Homogeneous Turbulence. Cambridge University Press, $212 \mathrm{pp}$.

Businger, J. A., and A. M. Yaglom, 1971: Introduction to Obukhov's paper on 'turbulence in an atmosphere with a non-uniform temperature.' Bound.-Layer Meteor., 2, 3-6, doi:10.1007/BF00718084.

—, J. C. Wyngaard, Z. Y. Izumi, and E. F. Bradley, 1971: Fluxprofile relationships in the atmospheric surface layer. J. Atmos. Sci., 28, 181-189, doi:10.1175/1520-0469(1971)028<0181: FPRITA $>2.0 . \mathrm{CO} ; 2$.
Chougule, A., 2013: Influence of atmospheric stability on the spatial structure of turbulence. Ph.D. thesis, Technical University of Denmark, $110 \mathrm{pp}$.

_ J. Mann, M. Kelly, J. Sun, D. H. Lenschow, and E. G. Patton, 2012: Vertical cross-spectral phases in neutral atmospheric flow. J. Turbul., 13, N36, doi:10.1080/14685248.2012.711524.

$\longrightarrow,-$ A. Segalini, and E. Dellwik, 2015: Spectral tensor parameters for wind turbine load modeling from forested and agricultural landscapes. Wind Energy, 18, 469-481, doi:10.1002/ we.1709.

de Maré, M., and J. Mann, 2014: Validation of the Mann spectral tensor for offshore wind conditions at different atmospheric stabilities. J. Phys.: Conf. Ser., 524, 012106, doi:10.1088/ 1742-6596/524/1/012106

$\longrightarrow$, and - 2016: On the space-time structure of sheared turbulence. Bound.-Layer Meteor., 160, 453-474, doi:10.1007/ s10546-016-0143-z.

Foken, T., 2006: 50 years of the Monin-Obukhov similarity theory. Bound.-Layer Meteor., 119, 431-447, doi:10.1007/s10546-006-9048-6.

Hanazaki, H., and J. C. R. Hunt, 1996: Linear processes in unsteady stably stratified turbulence. J. Fluid Mech., 318, 303-337, doi:10.1017/S0022112096007136.

— and —, 2004: Structure of unsteady stably stratified turbulence with mean shear. J. Fluid Mech., 507, 1-42, doi:10.1017/S0022112004007888.

Heidrick, T. R., S. Banerjee, and R. S. Azad, 1977: Experiments on the structure of turbulence in fully developed pipe flow: Interpretation of the measurements by a wave model. J. Fluid Mech., 81, 137-154, doi:10.1017/S0022112077001955.

Horst, T. W., J. Kleissl, D. H. Lenschow, C. Meneveau, C. H. Moeng, M. B. Parlange, P. P. Sullivan, and J. C. Weil, 2004: HATS: Field observations to obtain spatially filtered turbulence fields from crosswind arrays of sonic anemometers in the atmospheric surface layer. J. Atmos. Sci., 61, 1566-1581, doi:10.1175/1520-0469(2004)061<1566:HFOTOS > 2.0.CO;2.

Kaimal, J. C., and J. J. Finnigan, 1994: Atmospheric Boundary Layer Flows: Their Structure and Measurement. Oxford University Press, 289 pp.

— J. J. Wyngaard, Y. Izumi, and O. R. Coté, 1972: Spectral characteristics of surface-layer turbulence. Quart. J. Roy. Meteor. Soc., 98, 563-589, doi:10.1002/qj.49709841707.

Komori, S., H. Ueda, F. Ogino, and T. Mizushina, 1983: Turbulence structure in stably stratified open-channel flow. J. Fluid Mech., 130, 13-26, doi:10.1017/S0022112083000944.

Mann, J., 1994a: Models in micrometeorology. Ph.D. thesis, University of Aalborg, $127 \mathrm{pp}$.

_ 1994b: The spatial structure of neutral atmospheric surfacelayer turbulence. J. Fluid Mech., 273, 141-168, doi:10.1017/ S0022112094001886.

—, 1998: Wind field simulation. Probab. Eng. Mech., 13, 269-282, doi:10.1016/S0266-8920(97)00036-2.

- 2000: The spectral velocity tensor in moderately complex terrain. J. Wind Eng. Ind. Aerodyn., 88, 153-169, doi:10.1016/ S0167-6105(00)00046-5.

Maxey, M. R., 1982: Distortion of turbulence in flows with parallel streamlines. J. Fluid Mech., 124, 261-282, doi:10.1017/ S0022112082002493.

Obukhov, A. M., 1946: "Turbulentnost” v temperaturnoj neodnorodnoj atmosfere (Turbulence in an atmosphere with a nonuniform temperature). Tr. Inst. Theor. Geofiz. Akad. Nauk SSSR, 1, 95-115. , 1971: Turbulence in an atmosphere with a non-uniform temperature. Bound.-Layer Meteor., 2, 7-29, doi:10.1007/ BF00718085. 
Peña, A., S. Gryning, and J. Mann, 2010a: On the length-scale of the wind profile. Quart. J. Roy. Meteor. Soc., 136, 2119-2131, doi:10.1002/qj.714.

,$--\longrightarrow$, and C. B. Hasager, 2010b: Length scales of the neutral wind profile over homogeneous terrain. J. Appl. Meteor. Climatol., 49, 792-806, doi:10.1175/ 2009JAMC2148.1.

Pope, S. B., 2000: Turbulent Flows. 1st ed. Cambridge University Press, 802 pp.

Sathe, A., J. Mann, T. Barlas, W. A. A. M. Bierbooms, and G. J. W. van Bussel, 2013: Influence of atmospheric stability on wind turbine loads. Wind Energy, 16, 1013-1032, doi:10.1002/we.1528.

Segalini, A., and J. Arnqvist, 2015: A spectral model for stably stratified turbulence. J. Fluid Mech., 781, 330-352, doi:10.1017/ jfm.2015.502.
Sullivan, P. P., T. W. Horst, D. H. Lenschow, C. Moeng, and J. C. Weil, 2003: Structure of subfilter-scale fluxes in the atmospheric surface layer with application to large-eddy simulation modelling. J. Fluid Mech., 482, 101-139, doi:10.1017/ S0022112003004099.

Tennekes, H., and J. L. Lumley, 1972: A First Course in Turbulence. MIT Press, 320 pp.

Townsend, A. A., 1976: The Structure of Turbulent Shear Flow. 2nd ed. Cambridge University Press, 442 pp.

von Kármán, T., 1948: Progress in the statistical theory of turbulence. Proc. Natl. Acad. Sci. USA, 34, 530-539.

Wyngaard, J. C., 2010: Turbulence in the Atmosphere. Cambridge University Press, $406 \mathrm{pp}$

— spheric surface layer. Quart. J. Roy. Meteor. Soc., 98, 590-603, doi:10.1002/qj.49709841708. 